Research Article

\title{
On Ve-Degree-Based Irregularity Properties of the Crystallographic Structure of Molecules
}

\author{
Akbar Jahanbani (iD, Rana Khoeilar, and Hajar Shooshtari \\ Department of Mathematics, Azarbaijan Shahid Madani University, Tabriz, Iran \\ Correspondence should be addressed to Akbar Jahanbani; akbar.jahanbani92@gmail.com
}

Received 2 April 2021; Accepted 15 May 2021; Published 1 June 2021

Academic Editor: Marcelino Maneiro

Copyright (c) 2021 Akbar Jahanbani et al. This is an open access article distributed under the Creative Commons Attribution License, which permits unrestricted use, distribution, and reproduction in any medium, provided the original work is properly cited.

Irregularity indices are usually used for quantitative characterization of the topological structure of nonregular graphs. In numerous applications and problems in material engineering and chemistry, it is useful to be aware that how irregular a molecular structure is? In this paper, we are interested in formulating closed forms of irregularity measures of some of the crystallographic structures of $\mathrm{Cu}_{2} \mathrm{O}[p, q, r]$ and crystallographic structure of titanium difluoride of $T_{i} F_{2}[p, q, r]$. These theoretical conclusions provide practical guiding significance for pharmaceutical engineering and complex network and quantify the degree of folding of long organic molecules.

\section{Introduction}

In the medicines mathematical model, the structure of medication is taken as an undirected graph, where vertices and edges are taken as atoms and chemical bonds. Mathematical chemistry gives tools, for example, polynomials and numbers, to obtain properties of chemical compounds without utilizing quantum mechanics [1-3]. A topological index is a numerical parameter of a graph and describes its topology. It depicts the molecular structure numerically and is utilized in the advancement of qualitative structureactivity relationships (QSARs). There are three kinds of topological indices:

(1) Degree-based.

(2) Distance-based.

(3) Spectral-based.

In theoretical chemistry and biology, topological indices have been used for working out the information on molecules in the form of numerical coding. This relates to characterizing physicochemical, biological, toxicologic, pharmacologic, and other properties of chemical compounds. Thousands of molecular structure descriptors have been suggested in order to characterize the physical and chemical properties of molecules [4-6].

Degree-based indices can be further classified in the class of irregularity indices that measure the irregularity of the given graph. Recently, Réti et al. $[7,8]$ showed that the graph irregularity indices are efficient in quantitative structureproperty relationship (QSPR) studies of molecular graphs [9].

Let $\mathrm{Cu}_{2} \mathrm{O}[p, q, r]$ be the chemical graph of $\mathrm{Cu}_{2} \mathrm{O}$ with $p \times q$ unit cells in the plane and $r$ layers. Copper(I) oxide or cuprous oxide is the inorganic compound with the formula $\mathrm{Cu}_{2} \mathrm{O}$. Figure 1 describes the graph of the molecule $\mathrm{Cu}_{2} \mathrm{O}$ [10]. Note that copper atoms are shown in Figure 1 by red dots and oxygen atoms are shown by blue dots. In the $\mathrm{Cu}_{2} \mathrm{O}$ lattice graph, each copper atom is attached to two oxygen atoms, and every oxygen atom is attached to four copper atoms.

It is one of the principal oxides of copper, the other being $\mathrm{CuO}$ or cupric oxide. Nowadays, the crystallographic structure of the molecule $\mathrm{Cu}_{2} \mathrm{O}$ has attracted attention due to its interesting properties, low-cost, abundance, nontoxic nature [11]. This is the main reason to choose $\mathrm{Cu}_{2} \mathrm{O}$ and compute irregularity indices for it. Figures $1-5$ represent molecular graphs of these two systems. 


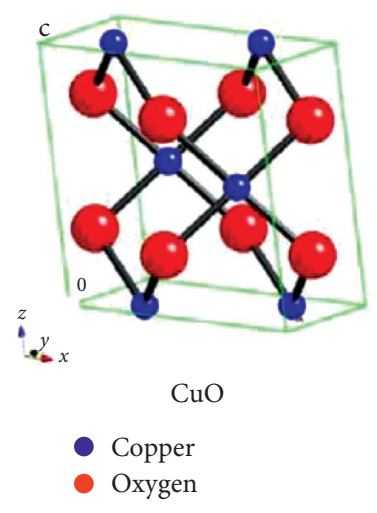

(a)

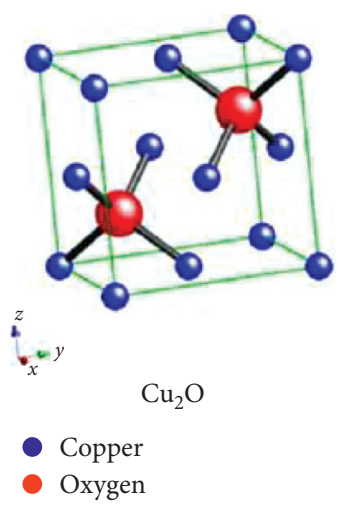

(b)

Figure 1: The structure of the molecule $\mathrm{Cu}_{2} \mathrm{O}$.

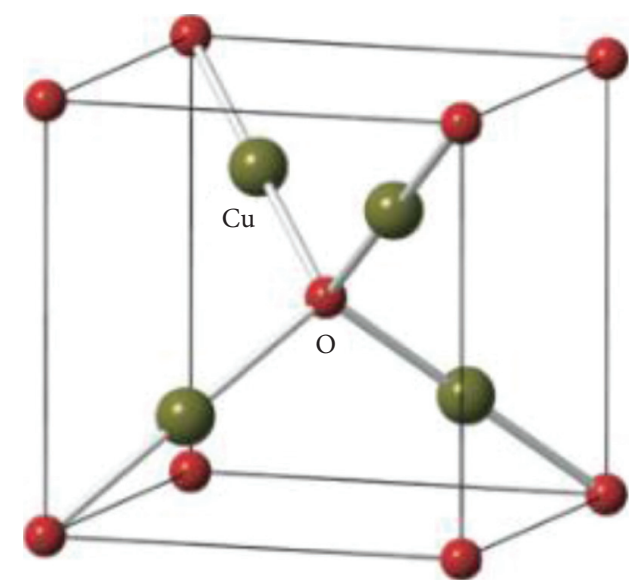

Figure 2: The graph of $\mathrm{Cu}_{2} \mathrm{O}[1,1,1]$.

Zahid et al. [12] computed the irregularity indices of a nanotube, Gao et al. [13] recently computed irregularity measures of some dendrimer structures, in [14], they had discussed irregularity molecular descriptors of hourglass, jagged-rectangle, and triangular benzenoid systems, Iqbal et al. [15] computed the irregularity indices of nanosheets, Zheng et al. [16] discussed irregularity measures of subdivision vertex-edge, Abdo et al. [17] computed irregularity of some molecular structures, Iqbal [12] studied irregularity measures of some nanotubes, and Gao et al. [18] obtained M-polynomials of the crystallographic structure of molecules. In [19], the authors investigated the total irregularity of trees with bounded maximal degree $\Delta$ and state integer linear programming problem which gives standard information about extremal trees. In many applications and problems, it is of importance to know how much a given graph deviates from being regular, i.e., how great its irregularity is [20]. Since then, irregular graphs and the degree of irregularity have become one of the core open problems of graph theory. In the current article, we are interested in finding the irregularity of the crystallographic structure of molecules
$\mathrm{Cu}_{2} \mathrm{O}$ and $T_{i} F_{2}[p, q, r]$ and computing and comparing the irregularities of some relevant chemical graphs.

Throughout this article, all graphs are finite, undirected, and simple. Let $G=(V(G), E(G))$ be such a graph with vertex set $V(G)$ and edge set $E(G)$. The order of $G$ is the cardinality of its vertex set, and size is the cardinality of its edge set. The vertices of $G$ correspond to atoms, and an edge between two vertices is related to the chemical bond between these vertices. The degree of a vertex $u$ of a graph $G$ is symbolized by $d_{G}(u)=d(u)$ and is defined as the number of edges incident with $u$. A graph is said to be regular if all its vertices have the same degree; otherwise, it is irregular. For details on the bases of graph theory, we refer to the book [21].

For a graph $G$, Albertson [22] defines the imbalance of an edge $e=u v \in E(G)$ as $|d G(u)-d G(v)|$ and the irregularity of $G$ as

$$
\operatorname{IRR}=\operatorname{IRR}(G)=\sum_{u v \in E(G)}|d(u)-d(v)| .
$$

For more information about the IRR $(G)$, you can see [23]. Abdo et al. [24] introduced the total irregularity index, as follows: 


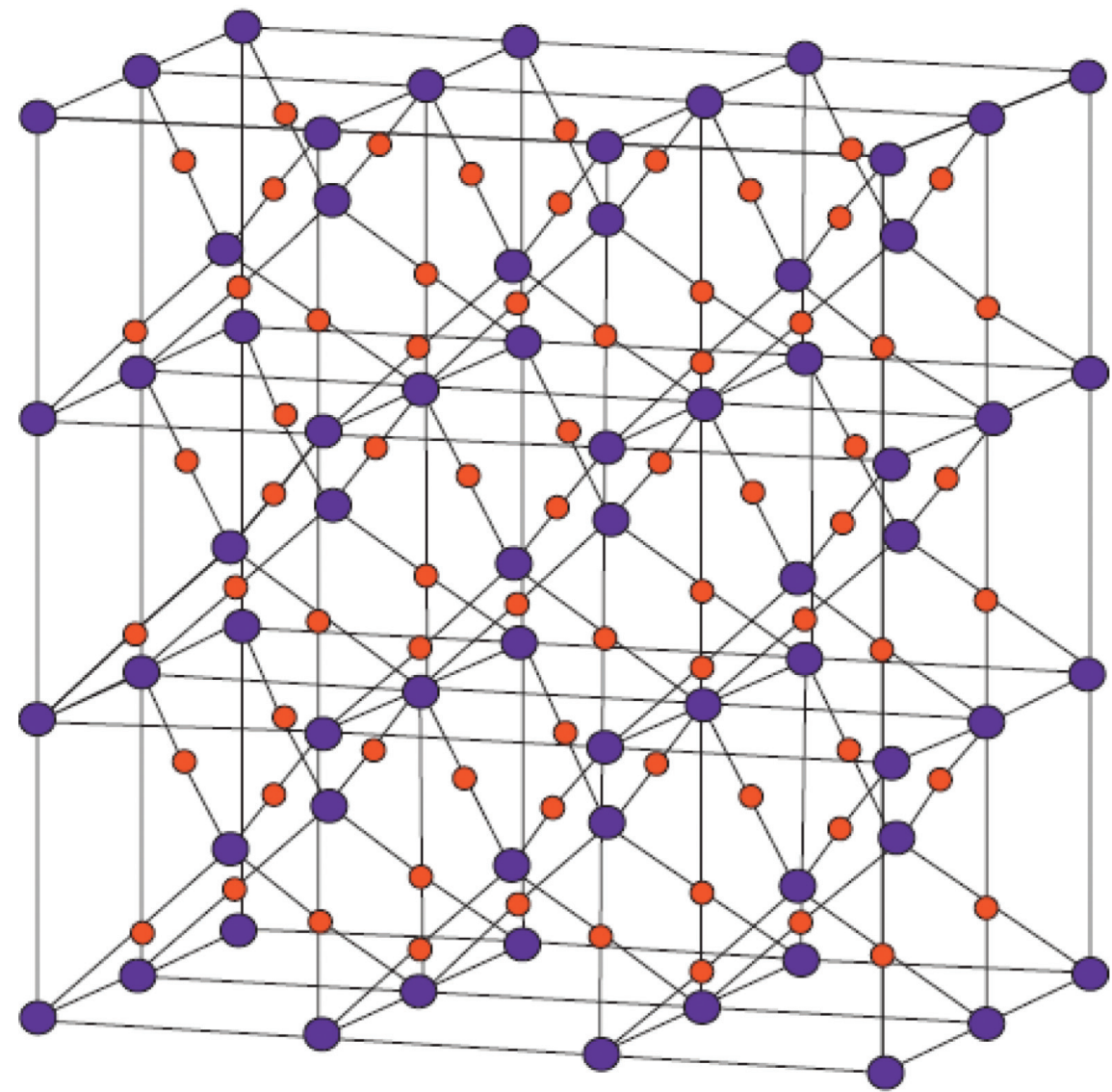

FIgURe 3: The graph of $\mathrm{Cu}_{2} \mathrm{O}[3,2,3]$.

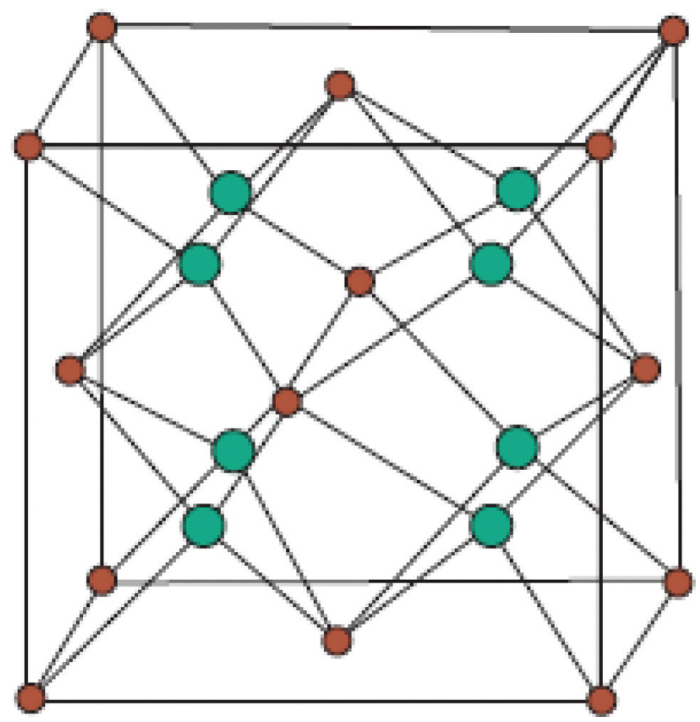

Figure 4: The graph of $T_{i} F_{2}[1,1,1]$.

$$
\operatorname{IRR}_{t}=\operatorname{IRR}_{t}(G)=\frac{1}{2} \sum_{u v \in E(G)}|d(u)-d(v)| .
$$

Gutman et al. [25] introduced the $\operatorname{IRF}(G)$ irregularity index of the graph $G$ as follows:

$$
\operatorname{IRF}=\operatorname{IRF}(G)=\sum_{u v \in E(G)}(d(u)-d(v))^{2} .
$$

Simplified ways of expressing the irregularities are irregularity indices. These irregularity indices have been 


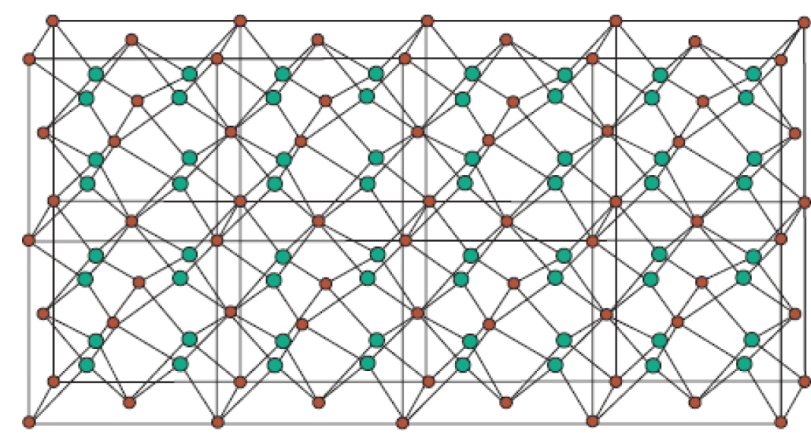

Figure 5: The graph of $T_{i} F_{2}[4,1,2]$.

studied recently in a novel way $[26,27]$. The first such irregularity index was introduced in [28]. Table 1 shows the rest of the irregularity indices used in this paper.

Most of the well-known degree-based irregularity indices can be obtained from the following general setting:

$$
\operatorname{IR}(G)=\sum_{u v \in E(G)} f(d(u), d(v))
$$

where $f(d(u), d(v))$ is an appropriately selected function.

\section{Results for the Crystallographic Structure}

In this section, we will compute the irregularity indices of some popular crystallographic structure of molecules $\mathrm{Cu}_{2} \mathrm{O}[p, q, r]$.

From [18], we know that the graph $\mathrm{Cu}_{2} \mathrm{O}[p, q, r]$ contains $6 p q r+p q+q r+p r+q+p+r+1$ vertices and $8 p q r$ edges.

We begin with obtaining formulas for irregularity measures of $\operatorname{IRR}\left(\mathrm{Cu}_{2} \mathrm{O}[p, q, r]\right), \operatorname{IRR}_{t}\left(\mathrm{Cu}_{2} \mathrm{O}[p, q, r]\right)$, and $\operatorname{IRF}\left(\mathrm{Cu}_{2} \mathrm{O}[p, q, r]\right)$.

Theorem 1. Let $C u_{2} O[p, q, r]$ be the crystallographic structure. Then, the irregularity indices of $\mathrm{Cu}_{2} \mathrm{O}[p, q, r]$ are

$$
\begin{aligned}
\operatorname{IRR}\left(\mathrm{Cu}_{2} \mathrm{O}[p, q, r]\right)= & 16 p q r-8 q r-8 p q-8 p r+12 p \\
& +12 q+12 r-16,
\end{aligned}
$$

$$
\begin{aligned}
\operatorname{IRR}_{t}\left(C u_{2} \mathrm{O}[p, q, r]\right)= & 8 p q r-4 q r-4 p q-4 p r+6 p \\
& +6 q+6 r-8, \\
\operatorname{IRF}\left(C u_{2} \mathrm{O}[p, q, r]\right)= & 32 p q r-16 q r-16 p q-16 p r \\
& +20 p+20 q+20 r-24 .
\end{aligned}
$$

Proof. Consider the graph crystallographic structure $\mathrm{Cu}_{2} \mathrm{O}[p, q, r]$. From the graph of $\mathrm{Cu}_{2} \mathrm{O}[p, q, r]$ crystallographic structure, we can see that there are three partitions, $V_{\{1\}}=\left\{v \in V\left(\mathrm{Cu}_{2} \mathrm{O}[p, q, r]\right) \mid d_{v}=1\right\}, \quad V_{\{2\}}=\left\{v \in V\left(\mathrm{Cu}_{2} \mathrm{O}\right.\right.$ $\left.[p, q, r]) \mid d_{v}=2\right\}$, and $V_{\{3\}}=\left\{v \in V\left(\mathrm{Cu}_{2} \mathrm{O}[p, q, r]\right) \mid d_{v}=4\right\}$. The edge set of $\mathrm{Cu}_{2} \mathrm{O}[p, q, r]$ can be partitioned as follows:

$$
\begin{aligned}
& E_{1}=\left\{e=u v \in E\left(\mathrm{Cu}_{2} \mathrm{O}[p, q, r]\right) \mid d(u)=1 \text { and } d(v)=2\right\}, \\
& E_{2}=\left\{e=u v \in E\left(\mathrm{Cu}_{2} \mathrm{O}[p, q, r]\right) \mid d(u)=2 \text { and } d(v)=2\right\}, \\
& E_{3}=\left\{e=u v \in E\left(\mathrm{Cu}_{2} \mathrm{O}[p, q, r]\right) \mid d(u)=2 \text { and } d(v)=4\right\} .
\end{aligned}
$$

From the molecular graph of $\mathrm{Cu}_{2} \mathrm{O}[p, q, r]$, we can observe in Figures $1-5$ that $\left|E_{1}\right|=4 p+4 q+4 r-$ $8,\left|E_{2}\right|=4 p q+4 p r+4 q r-8 p-8 q-8 r+12, \quad$ and $\left|E_{3}\right|=4(2 p q r-p q-p r-q r+p+q+r-1)$. Thus, by the definition of Albertson index for $\mathrm{Cu}_{2} \mathrm{O}[p, q, r]$, we have

$$
\begin{aligned}
\operatorname{IRR}\left(\mathrm{Cu}_{2} \mathrm{O}[p, q, r]\right) & =\sum_{u v \in E\left(\mathrm{Cu}_{2} \mathrm{O}[p, q, r]\right)}|d(u)-d(v)| \\
& =\sum_{u v \in E_{1}\left(\mathrm{Cu}_{2} \mathrm{O}[p, q, r]\right)} d(u)-d(v)+\sum_{u v \in E_{2}\left(\mathrm{Cu}_{2} \mathrm{O}[p, q, r]\right)}|d(u)-d(v)|+\sum_{u v \in E_{3}\left(\mathrm{Cu}_{2} \mathrm{O}[p, q, r]\right)} d(u)-d(v) \\
& =(4 p+4 q+4 r-8) \times|1-2|+4(2 p q r-p q-p r-q r+p+q+r-1) \times|2-4| \\
& =16 p q r-8 q r-8 p q-8 p r+12 p+12 q+12 r-16,
\end{aligned}
$$


TABLE 1: Some irregularity indices considered in [8].

\begin{tabular}{lc}
\hline Symbol & Irregularity indices \\
\hline IRDIF & The function $f(d(u), d(v))$ in equation $(28)$ \\
IRL & $(d(u) / d(v)-d(v) / d(u))$ \\
IRLU & $(\ln d(u)-\ln d(v))$ \\
IRLF & $|d(u)-d(v)| / \min (d(u), d(v))$ \\
IRLA & $|d(u)-d(v)| / \sqrt{(d(u) \times d(v))}$ \\
IRLD & $2|d(u)-d(v)| / d(u)+d(v)$ \\
IRGA & $\ln \{1+|d(u)-d(v)|\}$ \\
IRA & $\ln (|d(u)-d(v)| / \sqrt{(d(u) \times d(v))})$ \\
IRB & $\left(d(u)^{-1 / 2}-d(v)^{-1 / 2}\right)^{2}$ \\
\hline
\end{tabular}

which is the required formula result (5).

By the definition of total irregularity measure of $\mathrm{Cu}_{2} \mathrm{O}[p, q, r]$, we have

$$
\begin{aligned}
\operatorname{IRR}_{t}\left(\mathrm{Cu}_{2} \mathrm{O}[p, q, r]\right) & =\frac{1}{2} \sum_{u v \in E\left(\mathrm{Cu}_{2} \mathrm{O}[p, q, r]\right)}|d(u)-d(v)| \\
& =\frac{1}{2} \sum_{u v \in E\left(\mathrm{Cu}_{2} \mathrm{O}[p, q, r]\right)}|d(u)-d(v)|+\frac{1}{2} \sum_{u v \in E_{2}\left(\mathrm{Cu}_{2} \mathrm{O}[p, q, r]\right)}|d(u)-d(v)|+\frac{1}{2} \sum_{u v \in E_{3}\left(\mathrm{Cu}_{2} \mathrm{O}[p, q, r]\right)}|d(u)-d(v)| \\
& =\frac{1}{2} \times(4 p+4 q+4 r-8) \times|1-2|+\frac{1}{2} \times 4(2 p q r-p q-p r-q r+p+q+r-1) \times|2-4| \\
& =8 p q r-4 q r-4 p q-4 p r+6 p+6 q+6 r-8,
\end{aligned}
$$

which is the required formula result (7).

By the definition of irregularity index IRF for $\mathrm{Cu}_{2} \mathrm{O}[p, q, r]$, we have

$$
\begin{aligned}
\operatorname{IRF}\left(\mathrm{Cu}_{2} \mathrm{O}[p, q, r]\right) & =\sum_{u v \in E\left(\mathrm{Cu}_{2} \mathrm{O}[p, q, r]\right)}(d(u)-d(v))^{2} \\
& =\sum_{u v \in E_{1}\left(\mathrm{Cu}_{2} \mathrm{O}[p, q, r]\right)}(d(u)-d(v))^{2}+\sum_{u v \in E_{2}\left(\mathrm{Cu}_{2} \mathrm{O}[p, q, r]\right)}(d(u)-d(v))^{2}+\sum_{u v \in E_{3}\left(\mathrm{Cu}_{2} \mathrm{O}[p, q, r]\right)}(d(u)-d(v))^{2} \\
& =(4 p+4 q+4 r-8) \times(1-2)^{2}+4(2 p q r-p q-p r-q r+p+q+r-1) \times(2-4)^{2} \\
& =32 p q r-16 q r-16 p q-16 p r+20 p+20 q+20 r-24
\end{aligned}
$$

which is the required formula result (8).

Note that throughout the paper, colors red, blue, and green represent IRR, IRR

The next example represents some values of the calculated irregularity indices of $\operatorname{IRR}\left(\mathrm{Cu}_{2} \mathrm{O}[p, q, r]\right)$, $\mathrm{RR}_{t}\left(\mathrm{Cu}_{2} \mathrm{O}[p, q, r]\right)$, and $\operatorname{IRF}\left(\mathrm{Cu}_{2} \mathrm{O}[p, q, r]\right)$ for some test values of $p=q=r=1$.
Example 1. Let $\mathrm{Cu}_{2} \mathrm{O}[1,1,1]$ be crystallographic structure described in Figure 2. Then,

$$
\begin{aligned}
\operatorname{IRR}\left(\mathrm{Cu}_{2} \mathrm{O}[1,1,1]\right) & =12, \\
\operatorname{IRR}_{t}\left(\mathrm{Cu}_{2} \mathrm{O}[1,1,1]\right) & =6, \\
\operatorname{IRF}\left(\mathrm{Cu}_{2} \mathrm{O}[1,1,1]\right) & =20 .
\end{aligned}
$$


Theorem 2. Let $\mathrm{Cu}_{2} \mathrm{O}[p, q, r]$ be the crystallographic structure. Then, the irregularity indices of $\mathrm{Cu} \mathrm{u}_{2} \mathrm{O}[p, q, r]$ are

$$
\begin{aligned}
\operatorname{IRL}\left(\mathrm{Cu}_{2} \mathrm{O}[p, q, r]\right)= & 5.5451774444792 p q r-2.7725887222396 q r-2.7725887222396 p q-2.7725887222396 p r \\
& +5.5451774444792 p+5.5451774444792 q+5.54517744447927-8.3177661667188 \\
\operatorname{IRD}_{1}\left(\mathrm{Cu}_{2} \mathrm{O}[p, q, r]\right)= & 8.7888983093448 p q r-4.3944491546724 p q-4.3944491546724 p r-4.3944491546724 q r \\
& +7.167037876912 p+7.167037876912 q+7.167037876912 r-9.9396265991516 . \\
\operatorname{IRGA}\left(\mathrm{Cu}_{2} \mathrm{O}[p, q, r]\right)= & 1.8845285705024 p q r-0.9422642852512 p q-0.9422642852512 p r-0.9422642852512 q r \\
& +1.177830356564 p+1.177830356564 q+1.177830356564 r-0.7066982139384 .
\end{aligned}
$$

Proof. By definition of IRL irregularity index of $\mathrm{Cu}_{2} \mathrm{O}[p, q, r]$, we have

$$
\begin{aligned}
\operatorname{IRL}\left(\mathrm{Cu}_{2} \mathrm{O}[p, q, r]\right)= & \sum_{u v \in E\left(\mathrm{Cu}_{2} \mathrm{O}[p, q, r]\right)}|\ln d(u)-\ln d(v)| \\
= & \sum_{u v \in E_{1}\left(\mathrm{Cu}_{2} \mathrm{O}[p, q, r]\right)}|\ln d(u)-\ln d(v)|+\sum_{u v \in E_{2}} \sum_{\left(\mathrm{Cu}_{2} \mathrm{O}[p, q, r]\right)}|\ln d(u)-\ln d(v)| \\
& +\sum_{u v \in E_{3}\left(\mathrm{Cu}_{2} \mathrm{O}[p, q, r]\right)}|\ln d(u)-\ln d(v)| \\
= & (4 p+4 q+4 r-8) \times|\ln 1-\ln 2|+4(2 p q r-p q-p r-q r+p+q+r-1) \\
& \times|\ln 2-\ln 4|=5.5451774444792 p q r-2.7725887222396 q r-2.7725887222396 p q-2.7725887222396 p r \\
& +5.5451774444792 p+5.5451774444792 q+5.54517744447927 r-8.3177661667188 .
\end{aligned}
$$
have

By applying the definition of $\operatorname{IRD}_{1}$ for $\mathrm{Cu}_{2} \mathrm{O}[p, q, r]$, we

$$
\begin{aligned}
\operatorname{IRD}_{1}\left(\mathrm{Cu}_{2} \mathrm{O}[p, q, r]\right)= & \sum_{u v \in E\left(\mathrm{Cu}_{2} \mathrm{O}[p, q, r]\right)} \ln \{1+|d(u)-d(v)|\} \\
= & \sum_{u v \in E_{1}\left(\mathrm{Cu}_{2} \mathrm{O}[p, q, r]\right)} \ln \{1+|d(u)-d(v)|\} \\
& +\sum_{u v \in E_{2}\left(\mathrm{Cu}_{2} \mathrm{O}[p, q, r]\right)} \ln \{1+|d(u)-d(v)|\} \\
& +\sum_{u v \in E_{3}\left(\mathrm{Cu}_{2} \mathrm{O}[p, q, r]\right)} \ln \{1+|d(u)-d(v)|\} \\
= & (4 p+4 q+4 r-8) \times \ln \{1+|1-2|\}+4(2 p q r-p q-p r-q r+p+q+r-1) \times \ln \{1+|2-4|\} \\
= & 8.7888983093448 p q r-4.3944491546724 p q-4.3944491546724 p r-4.3944491546724 q r \\
& +7.167037876912 p+7.167037876912 q+7.167037876912 r-9.9396265991516 .
\end{aligned}
$$

Finally, by applying the definition of IRGA for $\mathrm{Cu}_{2} \mathrm{O}[p, q, r]$, we have 


$$
\begin{aligned}
\operatorname{IRGA}\left(\mathrm{Cu}_{2} \mathrm{O}[p, q, r]\right)= & \sum_{u v \in E\left(\mathrm{Cu}_{2} \mathrm{O}[p, q, r]\right)} \ln \left(\frac{d(u)+d(v)}{2 \sqrt{d(u) \times d(v)}}\right) \\
= & \sum_{u v \in E_{1}\left(\mathrm{Cu}_{2} \mathrm{O}[p, q, r]\right)} \ln \left(\frac{d(u)+d(v)}{2 \sqrt{d(u) \times d(v)}}\right)+\sum_{u v \in E_{2}\left(\mathrm{Cu}_{2} \mathrm{O}[p, q, r]\right)} \ln \left(\frac{d(u)+d(v)}{2 \sqrt{d(u) \times d(v)}}\right) \\
& +\sum_{u v \in E_{3}\left(\mathrm{Cu}_{2} \mathrm{O}[p, q, r]\right)} \ln \left(\frac{d(u)+d(v)}{2 \sqrt{d(u) \times d(v)}}\right) \\
= & (4 p+4 q+4 r-8) \times \ln \frac{1+2}{2 \sqrt{1 \times 2}}+(4 p q+4 p r+4 q r-8 p-8 q-8 r+12) \times \ln \frac{2+2}{2 \sqrt{2 \times 2}} \\
& +4(2 p q r-p q-p r-q r+p+q+r-1) \times \times \ln \frac{2+4}{2 \sqrt{2 \times 4}} \\
= & 1.8845285705024 p q r-0.9422642852512 p q-0.9422642852512 p r \\
& -0.9422642852512 q r+1.177830356564 p+1.177830356564 q+1.177830356564 r \\
& -0.7066982139384 .
\end{aligned}
$$

Note that throughout the paper, colors violet, yellow, and Peru represent IRL, IRD ${ }_{1}$, and IRGA, respectively.
Theorem 3. Let $\mathrm{Cu}_{2} \mathrm{O}[p, q, r]$ be the crystallographic structure. Then, the irregularity indices of $\mathrm{Cu}_{2} \mathrm{O}[p, q, r]$ are

$$
\begin{aligned}
\operatorname{IRA}\left(\mathrm{Cu}_{2} \mathrm{O}[p, q, r]\right) & =(4 p+4 q+4 r-8) \times\left(\frac{3}{2}-\sqrt{2}\right)+(2 p q r-p q-p r-q r+p+q+r-1) \times(3-2 \sqrt{2}), \\
\operatorname{IRB}\left(\mathrm{Cu}_{2} \mathrm{O}[p, q, r]\right) & =(4 p+4 q+4 r-8) \times(3-2 \sqrt{2})+(2 p q r-p q-p r-q r+p+q+r-1) \times(24-16 \sqrt{2}), \\
\operatorname{IRDIF}\left(\mathrm{Cu}_{2} \mathrm{O}[p, q, r]\right) & =12 p q r-6 q r-6 p q-6 p r+12 p+12 q+12 r-18 .
\end{aligned}
$$

Proof. By applying the definition of IRA for $\mathrm{Cu}_{2} \mathrm{O}[p, q, r]$, we have

$$
\begin{aligned}
\operatorname{IRA}\left(\mathrm{Cu}_{2} \mathrm{O}[p, q, r]\right)= & \sum_{u v \in E\left(\mathrm{Cu}_{2} \mathrm{O}[p, q, r]\right)}\left(\sqrt{\frac{1}{d(u)}}-\sqrt{\frac{1}{d(v)}}\right)^{2} \\
= & \sum_{u v \in E_{1}\left(\mathrm{Cu}_{2} \mathrm{O}[p, q, r]\right)}\left(\sqrt{\frac{1}{d(u)}}-\sqrt{\frac{1}{d(v)}}\right)^{2}+\sum_{u v \in E_{2}\left(\mathrm{Cu}_{2} \mathrm{O}[p, q, r]\right)}\left(\sqrt{\frac{1}{d(u)}}-\sqrt{\frac{1}{d(v)}}\right)^{2} \\
& +\sum_{u v \in E_{3}\left(\mathrm{Cu}_{2} \mathrm{O}[p, q, r]\right)}\left(\sqrt{\frac{1}{d(u)}}-\sqrt{\frac{1}{d(v)}}\right)^{2} \\
= & (4 p+4 q+4 r-8) \times\left(\sqrt{1}-\sqrt{\frac{1}{2}}\right)^{2}+4(2 p q r-p q-p r-q r+p+q+r-1) \times\left(\sqrt{\frac{1}{2}}-\sqrt{\frac{1}{4}}\right)^{2} \\
= & (4 p+4 q+4 r-8) \times\left(\frac{3}{2}-\sqrt{2}\right)+(2 p q r-p q-p r-q r+p+q+r-1) \times(3-2 \sqrt{2}) .
\end{aligned}
$$



have

By applying the definition of IRB for $\mathrm{Cu}_{2} \mathrm{O}[p, q, r]$, we

$$
\begin{aligned}
\operatorname{IRB}\left(\mathrm{Cu}_{2} \mathrm{O}[p, q, r]\right)= & \sum_{u v \in E\left(\mathrm{Cu}_{2} \mathrm{O}[p, q, r]\right)}(\sqrt{d(u)}-\sqrt{d(v)})^{2} \\
= & \sum_{u v \in E_{1}\left(\mathrm{Cu}_{2} \mathrm{O}[p, q, r]\right)}(\sqrt{d(u)}-\sqrt{d(v)})^{2} \\
& +\sum_{u v \in E_{2}\left(\mathrm{Cu}_{2} \mathrm{O}[p, q, r]\right)}(\sqrt{d(u)}-\sqrt{d(v)})^{2}+\sum_{u v \in E_{3}\left(\mathrm{Cu}_{2} \mathrm{O}[p, q, r]\right)}(\sqrt{d(u)}-\sqrt{d(v)})^{2} \\
= & (4 p+4 q+4 r-8) \times(\sqrt{1}-\sqrt{2})^{2}+4(2 p q r-p q-p r-q r+p+q+r-1) \times(\sqrt{2}-\sqrt{4})^{2} \\
= & (4 p+4 q+4 r-8) \times(3-2 \sqrt{2})+(2 p q r-p q-p r-q r+p+q+r-1) \times(24-16 \sqrt{2}) .
\end{aligned}
$$

Finally, by applying the definition of IRDIF for $\mathrm{Cu}_{2} \mathrm{O}[p, q, r]$, we have

$$
\begin{aligned}
\operatorname{IRDIF}\left(\mathrm{Cu}_{2} \mathrm{O}[p, q, r]\right) & =\sum_{u v \in E\left(\mathrm{Cu}_{2} \mathrm{O}[p, q, r]\right)}\left|\frac{d(u)}{d(v)}-\frac{d(v)}{d(u)}\right| \\
& =\sum_{u v \in E_{1}\left(\mathrm{Cu}_{2} \mathrm{O}[p, q, r]\right)}\left|\frac{d(u)}{d(v)}-\frac{d(v)}{d(u)}\right|+\sum_{u v \in E_{2}\left(\mathrm{Cu}_{2} \mathrm{O}[p, q, r]\right)}\left|\frac{d(u)}{d(v)}-\frac{d(v)}{d(u)}\right|+\sum_{u v \in E_{3}\left(\mathrm{Cu}_{2} \mathrm{O}[p, q, r]\right)}\left|\frac{d(u)}{d(v)}-\frac{d(v)}{d(u)}\right| \\
& =(4 p+4 q+4 r-8) \times\left|\frac{1}{2}-\frac{2}{1}\right|+4(2 p q r-p q-p r-q r+p+q+r-1) \times\left|\frac{2}{4}-\frac{4}{2}\right| \\
& =12 p q r-6 q r-6 p q-6 p r+12 p+12 q+12 r-18 .
\end{aligned}
$$

Note that throughout the paper, colors aquamarine, black, and blue violet represent IRA, IRB, and IRDIF, respectively.
Theorem 4. Let $\mathrm{Cu} u_{2} \mathrm{O}[p, q, r]$ be the crystallographic structure. Then, the irregularity indices of $\mathrm{Cu}{ }_{2} \mathrm{O}[p, q, r]$ are

$$
\begin{aligned}
& \operatorname{IRLU}\left(\mathrm{Cu}_{2} \mathrm{O}[p, q, r]\right)=8 p q r-4 q r-4 p q-4 p r+8 p+8 q+8 r-12, \\
& \operatorname{IRLA}\left(\mathrm{Cu}_{2} \mathrm{O}[p, q, r]\right)=\frac{8 p q r}{3}-\frac{4 q r}{3}-\frac{4 p q}{3}-\frac{4 p r}{3}+\frac{8 p}{3}+\frac{8 q}{3}+\frac{8 r}{3}-4, \\
& \operatorname{IRLF}\left(\mathrm{Cu}_{2} \mathrm{O}[p, q, r]\right)=(4 p+4 q+4 r-8) \times \sqrt{2}+(2 p q r-p q-p r-q r+p+q+r-1) \times 2 \sqrt{2} .
\end{aligned}
$$

Proof. By definition of IRLU irregularity index of $\mathrm{Cu}_{2} \mathrm{O}[p, q, r]$, we have 


$$
\begin{aligned}
\operatorname{IRLU}\left(\mathrm{Cu}_{2} \mathrm{O}[p, q, r]\right)= & \sum_{u v \in E\left(\mathrm{Cu}_{2} \mathrm{O}[p, q, r]\right)} \frac{|d(u)-d(v)|}{\min (d(u), d(v))} \\
= & \sum_{u v \in E_{1}\left(\mathrm{Cu}_{2} \mathrm{O}[p, q, r]\right)} \frac{|d(u)-d(v)|}{\min (d(u), d(v))}+\sum_{u v \in E_{2}\left(\mathrm{Cu}_{2} \mathrm{O}[p, q, r]\right)} \frac{|d(u)-d(v)|}{\min (d(u), d(v))} \\
& +\sum_{u v \in E_{3}\left(\mathrm{Cu}_{2} \mathrm{O}[p, q, r]\right)} \frac{|d(u)-d(v)|}{\min (d(u), d(v))} \\
= & (4 p+4 q+4 r-8) \times \frac{|1-2|}{\min (1,2)}+4(2 p q r-p q-p r-q r+p+q+r-1) \times \frac{|2-4|}{\min (2,4)} \\
= & 8 p q r-4 q r-4 p q-4 p r+8 p+8 q+8 r-12 .
\end{aligned}
$$
have

By applying the definition of IRLA for $\mathrm{Cu}_{2} \mathrm{O}[p, q, r]$, we

$$
\begin{aligned}
\operatorname{IRLA}\left(\mathrm{Cu}_{2} \mathrm{O}[p, q, r]\right)= & \sum_{u v \in E\left(\mathrm{Cu}_{2} \mathrm{O}[p, q, r]\right)} 2 \frac{|d(u)-d(v)|}{(d(u)+d(v))} \\
= & \sum_{u v \in E_{1}\left(\mathrm{Cu}_{2} \mathrm{O}[p, q, r]\right)} 2 \frac{|d(u)-d(v)|}{(d(u)+d(v))}+\sum_{u v \in E_{2}\left(\mathrm{Cu}_{2} \mathrm{O}[p, q, r]\right)} 2 \frac{|d(u)-d(v)|}{(d(u)+d(v))}+\sum_{u v \in E_{3}\left(\mathrm{Cu}_{2} \mathrm{O}[p, q, r]\right)} 2 \frac{|d(u)-d(v)|}{(d(u)+d(v))} \\
= & (4 p+4 q+4 r-8) \times \frac{|1-2|}{(1+2)}+4(2 p q r-p q-p r-q r+p+q+r-1) \times \frac{|2-4|}{(2+4)}=\frac{8 p q r}{3} \\
& -\frac{4 q r}{3}-\frac{4 p q}{3}-\frac{4 p r}{3}+\frac{8 p}{3}+\frac{8 q}{3}+\frac{8 r}{3}-4 .
\end{aligned}
$$

By applying the definition of IRLF for $\mathrm{Cu}_{2} \mathrm{O}[p, q, r]$, we have

$$
\begin{aligned}
\operatorname{IRLF}\left(\mathrm{Cu}_{2} \mathrm{O}[p, q, r]\right)= & \sum_{u v \in E\left(\mathrm{Cu}_{2} \mathrm{O}[p, q, r]\right)} \frac{|d(u)-d(v)|}{\sqrt{d(u) \times d(v)}} \\
= & \sum_{u v \in E_{1}\left(\mathrm{Cu}_{2} \mathrm{O}[p, q, r]\right)} \frac{|d(u)-d(v)|}{\sqrt{d(u) \times d(v)}}+\sum_{u v \in E_{2}\left(\mathrm{Cu}_{2} \mathrm{O}[p, q, r]\right)} \frac{|d(u)-d(v)|}{\sqrt{d(u) \times d(v)}}+\sum_{u v \in E_{3}\left(\mathrm{Cu}_{2} \mathrm{O}[p, q, r]\right)} \frac{|d(u)-d(v)|}{\sqrt{d(u) \times d(v)}} \\
= & (4 p+4 q+4 r-8) \times \frac{|1-2|}{\sqrt{1 \times 2}}+4(2 p q r-p q-p r-q r+p+q+r-1) \times \frac{|2-4|}{\sqrt{2 \times 4}} \\
= & (4 p+4 q+4 r-8) \times \sqrt{2} \\
& +(2 p q r-p q-p r-q r+p+q+r-1) \times 2 \sqrt{2} .
\end{aligned}
$$

Note that throughout the paper, colors dark orange, fuchsia, and gray represent IRLU, IRLA, and IRLF, respectively.
Next example represents some values of the calculated irregularity indices of $\operatorname{IRLU}\left(\mathrm{Cu}_{2} \mathrm{O}[p, q, r]\right)$, $\operatorname{IRLA}\left(\mathrm{Cu}_{2} \mathrm{O}[p, q, r]\right), \operatorname{IRLF}\left(\mathrm{Cu}_{2} \mathrm{O}[p, q, r]\right)$, and IRDIF 
$\left(\mathrm{Cu}_{2} \mathrm{O}[p, q, r]\right)$ for some test values of $p=3, q=2$, and $r=3$.

Example 2. Let $\mathrm{Cu}_{2} \mathrm{O}[3,2,3]$ be crystallographic structure described in Figure 3.

Then,

$$
\begin{aligned}
\operatorname{IRLU}\left(\mathrm{Cu}_{2} \mathrm{O}[3,2,3]\right) & =112, \\
\operatorname{IRLA}\left(\mathrm{Cu}_{2} \mathrm{O}[3,2,3]\right) & =37.3333333333333, \\
\operatorname{IRLF}\left(\mathrm{Cu}_{2} \mathrm{O}[3,2,3]\right) & =79.1959594928933, \\
\operatorname{IRDIF}\left(\mathrm{Cu}_{2} \mathrm{O}[3,2,3]\right) & =168 .
\end{aligned}
$$

\section{Results for the Crystallographic Structure of Titanium Difluoride}

In this section, we compute irregularity measures of crystal structure of titanium difluoride of $T_{i} F_{2}[p, q, r]$.
Titanium difluoride is a water-insoluble titanium hotspot for use in oxygen-delicate applications, for example, metal generation. The concoction chart of the crystallographic structure of $T_{i} F_{2}[p, q, r]$ is depicted in Figure 1 graph unit cell of $\mathrm{Cu}_{2} \mathrm{O}$ (see [29]). In Figures 4 and 5, red dots are for $F$ atoms and green dots are for $T_{i}$ atoms.

From [18], we know that the graph $T_{i} F_{2}[p, q, r]$ contains $12 p q r+2 p q+2 p r+2 q r+p+q+r+1$ vertices and $32 p q r$ edges.

Here, we obtain some formulas for irregularity measures of $\quad \operatorname{IRR}\left(T_{i} F_{2}[p, q, r]\right), \quad \operatorname{IRR}_{t}\left(T_{i} F_{2}[p, q, r]\right), \quad$ and $\operatorname{IRF}\left(T_{i} F_{2}[p, q, r]\right)$.

Theorem 5. Let $T_{i} F_{2}[p, q, r]$ be the crystallographic structure of titanium difluoride. Then, the irregularity indices of $T_{i} F_{2}[p, q, r]$ are

$$
\begin{aligned}
& \operatorname{IRR}\left(T_{i} F_{2}[p, q, r]\right)=128 p q r-64 q r-64 p q-64 p r+48 p+48 q+48 r-56, \\
& \operatorname{IRR}_{t}\left(T_{i} F_{2}[p, q, r]\right)=64 p q r-32 q r-32 p q-32 p r+24 p+24 q+24 r-28, \\
& \operatorname{IRF}\left(T_{i} F_{2}[p, q, r]\right)=512 p q r-256 q r-256 p q-256 p r+160 p+160 q+160 r-152 .
\end{aligned}
$$

Proof. Consider the graph crystallographic structure of titanium difluoride $T_{i} F_{2}[p, q, r]$. From the graph of $T_{i} F_{2}[p, q, r]$ crystallographic structure of titanium difluoride, we can see that there are four partitions, $V_{\{1\}}=\left\{v \in V\left(T_{i} F_{2}[p, q, r]\right) \mid d(v)=1\right\}$,
$V_{\{2\}}=\left\{v \in V\left(T_{i} F_{2}[p, q, r]\right) \mid d(v)=2\right\}, \quad V_{\{3\}}=\{v \in V$ $\left.\left(T_{i} F_{2}[p, q, r]\right) \mid d(v)=4\right\}$, and $V_{\{4\}}=\left\{v \in V\left(T_{i} F_{2}[p, q, r]\right) \mid\right.$ $d(v)=8\}$. The edge set of $T_{i} F_{2}[p, q, r]$ can be partitioned as follows:

$$
\begin{aligned}
& E_{1}=\left\{e=u v \in E\left(T_{i} F_{2}[p, q, r]\right) \mid d(u)=1 \text { and } d(v)=4\right\}, \\
& E_{2}=\left\{e=u v \in E\left(T_{i} F_{2}[p, q, r]\right) \mid d(u)=2 \text { and } d(v)=4\right\}, \\
& E_{3}=\left\{e=u v \in E\left(T_{i} F_{2}[p, q, r]\right) \mid d(u)=4 \text { and } d(v)=4\right\}, \\
& E_{4}=\left\{e=u v \in E\left(T_{i} F_{2}[p, q, r]\right) \mid d(u)=4 \text { and } d(v)=8\right\} .
\end{aligned}
$$

From the molecular graph of $T_{i} F_{2}[p, q, r]$, we can observe in Figures 4 and 5 that $\left|E_{1}\right|=8,\left|E_{2}\right|=8(p+q+r-3)$, $\left|E_{3}\right|=16(p q+p r+q r)-16(p+q+r)+24$, and $\left|E_{2}\right|=$
$32 p q r-16(p q+p r+q r)+8(p+q+r)-8$. By applying the definition of Albertson index for $T_{i} F_{2}[p, q, r]$, we have

$$
\begin{aligned}
\operatorname{IRR}\left(T_{i} F_{2}[p, q, r]\right)= & \sum_{u v \in E\left(T_{i} F_{2}[p, q, r]\right)}|d(u)-d(v)| \\
= & \sum_{u v \in E_{1}\left(T_{i} F_{2}[p, q, r]\right)}|d(u)-d(v)|+\sum_{u v \in E_{2}\left(T_{i} F_{2}[p, q, r]\right)}|d(u)-d(v)|+\sum_{u v \in E_{3}\left(T_{i} F_{2}[p, q, r]\right)}|d(u)-d(v)| \\
& +\sum_{u v \in E_{4}\left(T_{i} F_{2}[p, q, r]\right)}|d(u)-d(v)| \\
= & 8 \times|1-4|+8(p+q+r-3) \times|2-4|+(32 p q r-16(p q+p r+q r))+(8(p+q+r)-8) \times|4-8| \\
= & 128 p q r-64 q r-64 p q-64 p r+48 p+48 q+48 r-56,
\end{aligned}
$$


which is the required result (26). By applying the definition of total irregularity measure for $T_{i} F_{2}[p, q, r]$, we have

$$
\begin{aligned}
\operatorname{IRR}_{t}\left(\mathrm{Cu}_{2} \mathrm{O}[p, q, r]\right)= & \frac{1}{2} \sum_{u v \in E\left(T_{i} F_{2}[p, q, r]\right)}|d(u)-d(v)| \\
= & \frac{1}{2} \sum_{u v \in E_{1}\left(T_{i} F_{2}[p, q, r]\right)}|d(u)-d(v)|+\frac{1}{2} \sum_{u v \in E_{2}\left(T_{i} F_{2}[p, q, r]\right)}|d(u)-d(v)|+\frac{1}{2} \sum_{u v \in E_{3}\left(T_{i} F_{2}[p, q, r]\right)}|d(u)-d(v)| \\
& +\frac{1}{2} \sum_{u v \in E_{4}\left(T_{i} F_{2}[p, q, r]\right)}|d(u)-d(v)| \\
= & \frac{1}{2} \times 8 \times|1-4|+\frac{1}{2} \times 8(p+q+r-3) \times|2-4|+(32 p q r-16(p q+p r+q r)) \\
& +(8(p+q+r)-8) \times \frac{1}{2} \times|4-8| \\
= & 64 p q r-32 q r-32 p q-32 p r+24 p+24 q+24 r-28,
\end{aligned}
$$

which is the required result (27).

By applying the definition of IRF for $T_{i} F_{2}[p, q, r]$, we have

$$
\begin{aligned}
\operatorname{IRF}\left(T_{i} F_{2}[p, q, r]\right)= & \sum_{u v \in E\left(T_{i} F_{2}[p, q, r]\right)}(d(u)-d(v))^{2} \\
= & \sum_{u v \in E_{1}\left(T_{i} F_{2}[p, q, r]\right)}(d(u)-d(v))^{2}+\sum_{u v \in E_{2}\left(C T_{i} F_{2}[p, q, r]\right)}(d(u)-d(v))^{2}+\sum_{u v \in E_{3}\left(T_{i} F_{2}[p, q, r]\right)}(d(u)-d(v))^{2} \\
& +\sum_{u v \in E_{4}\left(T_{i} F_{2}[p, q, r]\right)}(d(u)-d(v))^{2} \\
= & 8 \times(1-4)^{2}+8(p+q+r-3) \times(2-4)^{2}+\left(32 p q r-16(p q+p r+q r)+(8(p+q+r)-8) \times(4-8)^{2}\right. \\
= & 512 p q r-256 q r-256 p q-256 p r+160 p+160 q+160 r-152,
\end{aligned}
$$

which is the required result (28).

The next example represents some values of the calculated irregularity indices of $\operatorname{IRAL}\left(T_{i} F_{2}[p, q, r]\right)$, $\operatorname{IRR}_{t}\left(T_{i} F_{2}[p, q, r]\right)$, and $\operatorname{IRF}\left(T_{i} F_{2}[p, q, r]\right)$ for some test values of $p=q=r=1$.

Example 3. Let $T_{i} F_{2}[1,1,1]$ be the crystallographic structure of titanium difluoride described in Figure 4. Then,

$$
\begin{aligned}
\operatorname{IRAL}\left(T_{i} F_{2}[1,1,1]\right) & =24, \\
\operatorname{IRR}_{t}\left(T_{i} F_{2}[1,1,1]\right) & =12, \\
\operatorname{IRF}\left(T_{i} F_{2}[1,1,1]\right) & =72 .
\end{aligned}
$$

Theorem 6. Let $T_{i} F_{2}[p, q, r]$ be the crystallographic structure of titanium difluoride. Then, the irregularity indices of $T_{i} F_{2}[p, q, r]$ are 


$$
\begin{aligned}
\operatorname{IRL}\left(T_{i} F_{2}[p, q, r]\right)= & 22.1807097779168 p q r-11.0903548889584 q r-11.0903548889584 p q-11.0903548889584 p r \\
& +11.0903548889584 p+11.0903548889584 q+11.0903548889584 r-11.0903548889576 \\
\operatorname{IRD}_{1}\left(T_{i} F_{2}[p, q, r]\right)= & 51.5020131978912 p q r-25.7510065989456 p q-25.7510065989456 p r-25.7510065989456 q r \\
& +21.6644016088176 p+21.6644016088176 q+21.6644016088176 r-28.151843338548 \\
\operatorname{IRGA}\left(T_{i} F_{2}[p, q, r]\right)= & 1.8845285705024 p q r-0.9422642852512 p q-0.9422642852512 p r-0.9422642852512 q r \\
& +1.177830356564 p+1.177830356564 q+1.177830356564 r-0.0993801599888 .
\end{aligned}
$$

Proof. By applying the definition of IRL for $T_{i} F_{2}[p, q, r]$, we have

$$
\begin{aligned}
\operatorname{IRL}\left(T_{i} F_{2}[p, q, r]\right)= & \sum_{u v \in E\left(T_{i} F_{2}[p, q, r]\right)}|\ln d(u)-\ln d(v)| \\
= & \sum_{u v \in E_{1}\left(T_{i} F_{2}[p, q, r]\right)}|\ln d(u)-\ln d(v)|+\sum_{u v \in E_{2}\left(T_{i} F_{2}[p, q, r]\right)}|\ln d(u)-\ln d(v)| \\
& +\sum_{u v \in E_{3}\left(T_{i} F_{2}[p, q, r]\right)}|\ln d(u)-\ln d(v)| \\
= & 8 \times|\ln 1-\ln 4|+8(p+q+r-3) \times|\ln 2-\ln 4|+(32 p q r-16(p q+p r+q r \\
& +(8(p+q+r))-8) \times|\ln 4-\ln 8| \\
= & 22.1807097779168 p q r-11.0903548889584 q r-11.0903548889584 p q-11.0903548889584 p r \\
& +11.0903548889584 p+11.0903548889584 q+11.0903548889584 r-11.0903548889576 .
\end{aligned}
$$

By applying the definition of $\operatorname{IRD}_{1}$ for $T_{i} F_{2}[p, q, r]$, we have

$$
\begin{aligned}
\operatorname{IRD}_{1}\left(T_{i} F_{2}[p, q, r]\right)= & \sum_{u v \in E\left(T_{i} F_{2}[p, q, r]\right)} \ln \{1+|d(u)-d(v)|\} \\
= & \sum_{u v \in E_{1}\left(T_{i} F_{2}[p, q, r]\right)} \ln \{1+|d(u)-d(v)|\}+\sum_{u v \in E_{2}\left(T_{i} F_{2}[p, q, r]\right)} \ln \{1+|d(u)-d(v)|\} \\
& +\sum_{u v \in E_{3}\left(T_{i} F_{2}[p, q, r]\right)} \ln \{1+|d(u)-d(v)|\}+\sum_{u v \in E_{3}\left(T_{i} F_{2}[p, q, r]\right)} \ln \{1+|d(u)-d(v)|\} \\
= & 8 \times \ln \{1+|1-4|\}+8(p+q+r-3) \times \ln \{1+|2-4|\} \\
& +(32 p q r-16(p q+p r+q r))+(8(p+q+r)-8) \times \ln \{1+|4-8|\} \\
= & 51.5020131978912 p q r-25.7510065989456 p q-25.7510065989456 p r \\
& -25.7510065989456 q r+21.6644016088176 p+21.6644016088176 q \\
& +21.6644016088176 r-28.151843338548 .
\end{aligned}
$$


Finally, by applying the definition of IRGA for $T_{i} F_{2}[p, q, r]$, we have

$$
\begin{aligned}
\operatorname{IRGA}\left(T_{i} F_{2}[p, q, r]\right)= & \sum_{u v \in E\left(T_{i} F_{2}[p, q, r]\right)} \ln \frac{d(u)+d(v)}{2 \sqrt{d(u) \times d(v)}} \\
= & \sum_{u v \in E_{1}\left(T_{i} F_{2}[p, q, r]\right)} \ln \frac{d(u)+d(v)}{2 \sqrt{d(u) \times d(v)}}+\sum_{u v \in E_{2}\left(T_{i} F_{2}[p, q, r]\right)} \ln \frac{d(u)+d(v)}{2 \sqrt{d(u) \times d(v)}} \\
& +\sum_{u v \in E_{3}\left(T_{i} F_{2}[p, q, r]\right)} \ln \frac{d(u)+d(v)}{2 \sqrt{d(u) \times d(v)}}+\sum_{u v \in E_{4}\left(T_{i} F_{2}[p, q, r]\right)} \ln \frac{d(u)+d(v)}{2 \sqrt{d(u) \times d(v)}} \\
= & 8 \times \ln \frac{1+4}{2 \sqrt{1 \times 4}}+8(p+q+r-3) \times \ln \frac{2+4}{2 \sqrt{2 \times 4}}+(16 p q+16 p r+16 q r-16 p-16 q-16 r+24) \times \\
& \times \ln \frac{4+4}{2 \sqrt{4 \times 4}}+(32 p q r-16(p q+p r+q r)+(8(p+q+r-8) \times) \\
\ln \frac{4+8}{2 \sqrt{4 \times 8}}= & 1.8845285705024 p q r-0.9422642852512 p q-0.9422642852512 p r-0.9422642852512 q r+1.177830356564 p \\
& +1.177830356564 q+1.177830356564 r-0.0993801599888 .
\end{aligned}
$$

Theorem 7. Let $T_{i} F_{2}[p, q, r]$ be the crystallographic structure of titanium difluoride. Then, the irregularity indices of $T_{i} F_{2}[p, q, r]$ are

$$
\begin{aligned}
\operatorname{IRA}\left(T_{i} F_{2}[p, q, r]\right) & =(4 p+4 q+4 r-8) \times(6-4 \sqrt{2})+(2 p q r-p q-p r-q r+p+q+r-1) \times\left(-\frac{\sqrt{2}}{4}+\frac{3}{8}\right)+2 \\
\operatorname{IRB}\left(T_{i} F_{2}[p, q, r]\right) & =(4 p+4 q+4 r-8) \times(48-32 \sqrt{2})+(2 p q r-p q-p r-q r+p+q+r-1) \times(12-8 \sqrt{2})+8 \\
\operatorname{IRDIF}\left(T_{i} F_{2}[p, q, r]\right) & =48 p q r-24 q r-24 p q-24 p r+24 p+24 q+24 r-18
\end{aligned}
$$

Proof. By applying the definition of IRA for $T_{i} F_{2}[p, q, r]$, we have 


$$
\begin{aligned}
\operatorname{IRA}\left(T_{i} F_{2}[p, q, r]\right)= & \sum_{u v \in E\left(T_{i} F_{2}[p, q, r]\right)}\left(\sqrt{\frac{1}{d(u)}}-\sqrt{\frac{1}{d(v)}}\right)^{2} \\
= & \sum_{u v \in E_{1}\left(T_{i} F_{2}[p, q, r]\right)}\left(\sqrt{\frac{1}{d(u)}}-\sqrt{\frac{1}{d(v)}}\right)^{2}+\sum_{u v \in E_{2}\left(T_{i} F_{2}[p, q, r]\right)}\left(\sqrt{\frac{1}{d(u)}}-\sqrt{\frac{1}{d(v)}}\right)^{2} \\
& +\sum_{u v \in E_{3}\left(T_{i} F_{2}[p, q, r]\right)}\left(\sqrt{\frac{1}{d(u)}}-\sqrt{\frac{1}{d(v)}}\right)^{2}+\sum_{u v \in E_{4}\left(T_{i} F_{2}[p, q, r]\right)}\left(\sqrt{\frac{1}{d(u)}}-\sqrt{\frac{1}{d(v)}}\right)^{2} \\
= & 8 \times\left(\sqrt{1}-\sqrt{\frac{1}{4}}\right)^{2}+8(p+q+r-3) \times\left(\sqrt{\frac{1}{2}}-\sqrt{\frac{1}{4}}\right)^{2}+(32 p q r-16(p q+p r+(p+q q r))+(8+r-8) \\
& \times\left(\sqrt{\frac{1}{4}}-\sqrt{\frac{1}{8}}\right)^{2} \\
= & (4 p+4 q+4 r-8) \times(6-4 \sqrt{2})+(2 p q r-p q-p r-q r+p+q+r-1) \times\left(-\frac{\sqrt{2}}{4}+\frac{3}{8}\right)+2 .
\end{aligned}
$$
have

By applying the definition of IRB for $T_{i} F_{2}[p, q, r]$, we

$$
\begin{aligned}
\operatorname{IRB}\left(T_{i} F_{2}[p, q, r]\right)= & \sum_{u v \in E\left(T_{i} F_{2}[p, q, r]\right)}(\sqrt{d(u)}-\sqrt{d(v)})^{2} \\
= & \sum_{u v \in E_{1}\left(T_{i} F_{2}[p, q, r]\right)}(\sqrt{d(u)}-\sqrt{d(v)})^{2}+\sum_{u v \in E_{2}\left(T_{i} F_{2}[p, q, r]\right)}(\sqrt{d(u)}-\sqrt{d(v)})^{2} \\
& +\sum_{u v \in E_{3}\left(T_{i} F_{2}[p, q, r]\right)}(\sqrt{d(u)}-\sqrt{d(v)})^{2}+\sum_{u v \in E_{3}\left(T_{i} F_{2}[p, q, r]\right)}(\sqrt{d(u)}-\sqrt{d(v)})^{2} \\
= & 8 \times(\sqrt{1}-\sqrt{4})^{2}+8(p+q+r-3) \times(\sqrt{2}-\sqrt{4})^{2}+(32 p q r-16(p q+p r+q r) \\
& +(8(p+q+r-8)) \times(\sqrt{4}-\sqrt{8})^{2} \\
= & (4 p+4 q+4 r-8) \times(48-32 \sqrt{2})+(2 p q r-p q-p r-q r+p+q+r-1) \times(12-8 \sqrt{2})+8 .
\end{aligned}
$$
have

By applying the definition of IRDIF for $T_{i} F_{2}[p, q, r]$, we 


$$
\begin{aligned}
\operatorname{IRDIF}\left(T_{i} F_{2}[p, q, r]\right)= & \sum_{u v \in E\left(T_{i} F_{2}[p, q, r]\right)}\left|\frac{d(u)}{d(v)}-\frac{d(v)}{d(u)}\right| \\
= & \sum_{u v \in E_{1}\left(T_{i} F_{2}[p, q, r]\right)}\left|\frac{d(u)}{d(v)}-\frac{d(v)}{d(u)}\right|+\sum_{u v \in E_{2}\left(T_{i} F_{2}[p, q, r]\right)}\left|\frac{d(u)}{d(v)}-\frac{d(v)}{d(u)}\right|+\sum_{u v \in E_{3}\left(T_{i} F_{2}[p, q, r]\right)}\left|\frac{d(u)}{d(v)}-\frac{d(v)}{d(u)}\right| \\
& +\sum_{u v \in E_{4}\left(T_{i} F_{2}[p, q, r]\right)}\left|\frac{d(u)}{d(v)}-\frac{d(v)}{d(u)}\right| \\
= & 8 \times\left|\frac{1}{4}-\frac{4}{1}\right|+8(p+q+r-3) \times\left|\frac{2}{4}-\frac{4}{2}\right|+(32 p q r-16(p q+p r+(p+q q r)))+(8+r-8) \times\left|\frac{4}{8}-\frac{8}{4}\right| \\
= & 48 p q r-24 q r-24 p q-24 p r+24 p+24 q+24 r-18 .
\end{aligned}
$$

Theorem 8. Let $T_{i} F_{2}[p, q, r]$ be the crystallographic structure of titanium difluoride. Then, the irregularity indices of $T_{i} F_{2}[p, q, r]$ are

$$
\begin{aligned}
\operatorname{IRLU}\left(T_{i} F_{2}[p, q, r]\right)= & 32 p q r-16 q r-16 p q-16 p r \\
& +16 p+16 q+16 r-8, \\
\operatorname{IRLA}\left(T_{i} F_{2}[p, q, r]\right)= & \frac{32 p q r}{3}-\frac{16 q r}{3}-\frac{16 p q}{3}-\frac{16 p r}{3} \\
& +\frac{16 p}{3}+\frac{16 q}{3}+\frac{16 r}{3}-8,
\end{aligned}
$$

Proof. By applying the definition of IRLU for $T_{i} F_{2}[p, q, r]$, we have

$$
\begin{aligned}
\operatorname{IRLF}\left(T_{i} F_{2}[p, q, r]\right)= & (p+q+r-3) \times 4 \sqrt{2} \\
& +(16 p q r-8 p q-8 p r-8 q r+4 p \\
& +4 q+4 r-4) \times \sqrt{2}+4 \sqrt{2} .
\end{aligned}
$$

$$
\begin{aligned}
\operatorname{IRLU}\left(T_{i} F_{2}[p, q, r]\right)= & \sum_{u v \in E\left(T_{i} F_{2}[p, q, r]\right)} \frac{|d(u)-d(v)|}{\min (d(u), d(v))} \\
= & \sum_{u v \in E_{1}\left(T_{i} F_{2}[p, q, r]\right)} \frac{|d(u)-d(v)|}{\min (d(u), d(v))}+\sum_{u v \in E_{2}\left(T_{i} F_{2}[p, q, r]\right)} \frac{|d(u)-d(v)|}{\min (d(u), d(v))}+\sum_{u v \in E_{3}\left(T_{i} F_{2}[p, q, r]\right)} \frac{|d(u)-d(v)|}{\min (d(u), d(v))} \\
& +\sum_{u v \in E_{4}\left(T_{i} F_{2}[p, q, r]\right)} \frac{|d(u)-d(v)|}{\min (d(u), d(v))} \\
= & 8 \times \frac{|1-4|}{\min (1,4)}+8(p+q+r-3) \times \frac{|2-4|}{\min (2,4)}+(32 p q r-16(p q+p r+(p+q q r) \\
& +(8+r-8) \times \frac{|4-8|}{\min (4,8)} \\
= & 32 p q r-16 q r-16 p q-16 p r+16 p+16 q+16 r-8
\end{aligned}
$$

which is the required result (42).By applying the definition of IRLA for $T_{i} F_{2}[p, q, r]$, we have 
TABLE 2: Comparison of IRR, $\mathrm{IRR}_{t}$, and IRF for crystallographic structure $\mathrm{Cu}_{2} \mathrm{O}[p, q, r]$.

\begin{tabular}{lccc}
\hline$[p, q, r]$ & IRR & $\operatorname{IRR}_{t}$ & \\
\hline$[1,1,1]$ & 12 & 6 & 20 \\
{$[2,2,2]$} & 88 & 44 & 160 \\
{$[3,3,3]$} & 308 & 154 & 588 \\
{$[4,4,4]$} & 768 & 384 & 1496 \\
\hline
\end{tabular}

TABLE 3: Comparison of IRL, $\mathrm{IRD}_{1}$, and IRGA for crystallographic structure $\mathrm{Cu}_{2} \mathrm{O}[p, q, r]$.

\begin{tabular}{lccc}
\hline$[p, q, r]$ & IRL & IRD $_{1}$ & IRGA \\
\hline$[1,1,1]$ & 5.5451774444792 & 7.167037876912 & 0.4711321426255 \\
{$[2,2,2]$} & 36.0436533891148 & 50.64039728101 & 3.062358927066 \\
{$[3,3,3]$} & 116.4487263340632 & 173.2138414692112 & 9.8937749951362 \\
{$[4,4,4]$} & 280.0314609461996 & 427.6207602975844 & 23.7921732025895 \\
\hline
\end{tabular}

$$
\begin{aligned}
& \operatorname{IRLA}\left(T_{i} F_{2}[p, q, r]\right)=\sum_{u v \in E\left(T_{i} F_{2}[p, q, r]\right)} 2 \frac{|d(u)-d(v)|}{(d(u)+d(v))} \\
& =\sum_{u v \in E_{1}\left(T_{i} F_{2}[p, q, r]\right)} 2 \frac{|d(u)-d(v)|}{(d(u)+d(v))}+\sum_{u v \in E_{2}\left(T_{i} F_{2}[p, q, r]\right)} 2 \frac{|d(u)-d(v)|}{(d(u)+d(v))}+\sum_{u v \in E_{3}\left(T_{i} F_{2}[p, q, r]\right)} 2 \frac{|d(u)-d(v)|}{(d(u)+d(v))} \\
& +\sum_{u v \in E_{4}\left(T_{i} F_{2}[p, q, r]\right)} 2 \frac{|d(u)-d(v)|}{(d(u)+d(v))} \\
& =8 \times \frac{|1-2|}{(1+2)}+8(p+q+r-3) \times \frac{|2-4|}{(2+4)}+\left(32 p q r-16\left(p q+p r+(p+q q r)+(8+r-8) \times \frac{|4-8|}{(4+8)}\right.\right. \\
& =\frac{32 p q r}{3}-\frac{16 q r}{3}-\frac{16 p q}{3}-\frac{16 p r}{3}+\frac{16 p}{3}+\frac{16 q}{3}+\frac{16 r}{3}-8 \text {, }
\end{aligned}
$$

which is the required result (43).

By applying the definition of IRLF for $T_{i} F_{2}[p, q, r]$, we have

$$
\begin{aligned}
\operatorname{IRLF}\left(T_{i} F_{2}[p, q, r]\right)= & \sum_{u v \in E\left(T_{i} F_{2}[p, q, r]\right)} \frac{|d(u)-d(v)|}{\sqrt{d(u) \times d(v)}} \\
= & \sum_{u v \in E_{1}\left(T_{i} F_{2}[p, q, r]\right)} \frac{|d(u)-d(v)|}{\sqrt{d(u) \times d(v)}}+\sum_{u v \in E_{2}\left(T_{i} F_{2}[p, q, r]\right)} \frac{|d(u)-d(v)|}{\sqrt{d(u) \times d(v)}+} \sum_{u v \in E_{3}\left(T_{i} F_{2}[p, q, r]\right)} \frac{|d(u)-d(v)|}{\sqrt{d(u) \times d(v)}} \\
& +\sum_{u v \in E_{4}\left(T_{i} F_{2}[p, q, r]\right)} \frac{|d(u)-d(v)|}{\sqrt{d(u) \times d(v)}} \\
= & 8 \times \frac{|1-2|}{\sqrt{1 \times 2}+8(p+q+r-3) \times \times \frac{|2-4|}{\sqrt{2 \times 4}}+\left(32 p q r-16\left(p q+p r+(p+q q r)+(8+r-8) \times \frac{|4-8|}{\sqrt{4 \times 8}}\right.\right.} \\
= & (p+q+r-3) \times 4 \sqrt{2}+(16 p q r-8 p q-8 p r-8 q r+4 p+4 q+4 r-4) \times \sqrt{2}+4 \sqrt{2},
\end{aligned}
$$


TABLE 4: Comparison of IRA, IRB, and IRDIF for crystallographic structure $\mathrm{Cu}_{2} \mathrm{O}[p, q, r]$.

\begin{tabular}{cccc}
\hline$[p, q, r]$ & IRA & IRB & IRDIF \\
\hline$[1,1,1]$ & 0.5147186257614 & 2.0588745030457 & 12 \\
{$[2,2,2]$} & 2.9167388793148 & 15.0984130223353 & 78 \\
{$[3,3,3]$} & 10.4659453904824 & 52.8444455781734 & 252 \\
{$[4,4,4]$} & 17.6720061511424 & 131.7679681949257 & 606 \\
\hline
\end{tabular}

TABLE 5: Comparison of IRLU, IRLA, and IRLF for crystallographic structure $\mathrm{Cu}_{2} \mathrm{O}[p, q, r]$.

\begin{tabular}{lccc}
\hline$[p, q, r]$ & IRLU & IRLA & IRLF \\
\hline$[1,1,1]$ & 8 & 2.0588745030457 & 16.9705627484771 \\
{$[2,2,2]$} & 52 & 17.3333333333333 & 124.4507934888324 \\
{$[3,3,3]$} & 168 & 56 & 435.5777772109133 \\
{$[4,4,4]$} & 404 & 34.6666666666667 & 1086.1160159025371 \\
\hline
\end{tabular}

TABLE 6: Comparison of IRR, $\operatorname{IRR}_{t}$, and IRF for crystallographic structure of titanium difluoride $T_{i} F_{2}[p, q, r]$.

\begin{tabular}{lccc}
\hline$[p, q, r]$ & IRR & IRR $_{t}$ & \\
\hline$[1,1,1]$ & 24 & 12 & 72 \\
{$[2,2,2]$} & 488 & 244 & 1832 \\
{$[3,3,3]$} & 2104 & 1052 & 8200 \\
{$[4,4,4]$} & 5640 & 2820 & 22248 \\
\hline
\end{tabular}

TABLE 7: Comparison of IRL, $\operatorname{IRD}_{1}$, and IRGA for the crystallographic structure of titanium difluoride $T_{i} F_{2}[p, q, r]$.

\begin{tabular}{lccc}
\hline$[p, q, r]$ & IRL & IRD $_{1}$ & IRGA \\
\hline$[2,2,2]$ & 83.1776616671888 & 165.8345663143578 & 69.554205551518 \\
{$[3,3,3]$} & 363.2091226133884 & 598.82777711408108 & 440.3809984072711 \\
{$[4,4,4]$} & 975.95123022834 & 1511.8209759672638 & 1291.2077912630243 \\
\hline
\end{tabular}

TABLE 8: Comparison of IRA, IRB, and IRDIF for the crystallographic structure of titanium difluoride $T_{i} F_{2}[p, q, r]$.

\begin{tabular}{lccc}
\hline$[p, q, r]$ & IRA & IRB & IRDIF \\
\hline$[1,1,1]$ & 3.3940296114372 & 19.6669555172591 & 12 \\
{$[2,2,2]$} & 7.6833514927824 & 58.0992795741124 & 78 \\
{$[3,3,3]$} & 12.3587123434488 & 108.8848506492402 & 252 \\
{$[4,4,4]$} & 17.6774714763168 & 180.259166754825 & \\
\hline
\end{tabular}

TABLE 9: Comparison of IRLU, IRLA, and IRLF for the crystallographic structure of titanium difluoride $T_{i} F_{2}[p, q, r]$.

\begin{tabular}{lccc}
\hline$[p, q, r]$ & IRLU & IRLA & IRLF \\
\hline$[1,1,1]$ & 24 & 2.6666666666667 & 0 \\
{$[2,2,2]$} & 152 & 45.33333333333333 & 184 \\
{$[3,3,3]$} & 568 & 482.6666666666667 & 90.5096679918781 \\
{$[4,4,4]$} & 1464 & & 84.6660889654819 \\
\hline
\end{tabular}

which is the required formula result (44).

Finally, the next example represents some values of the calculated irregularity indices of $\operatorname{IRLU}\left(T_{i} F_{2}[p, q, r]\right)$,
$\operatorname{IRLA}\left(T_{i} F_{2}[p, q, r]\right), \quad \operatorname{IRLF}\left(T_{i} F_{2}[p, q, r]\right), \quad$ and $\operatorname{IRDIF}\left(T_{i} F_{2}[p, q, r]\right)$ for some test values of $p=4, q=1$, and $r=2$. 


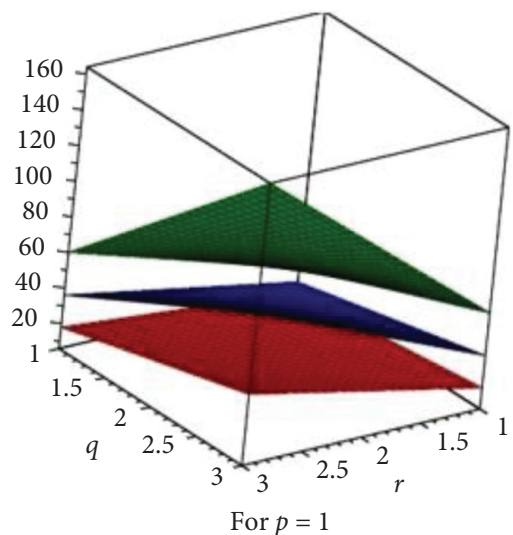

(a)

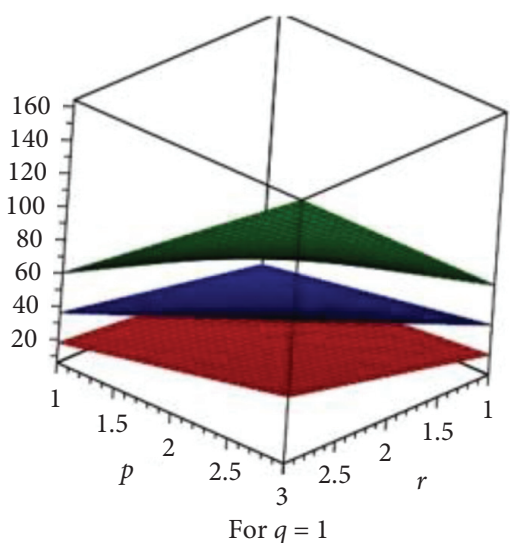

(b)

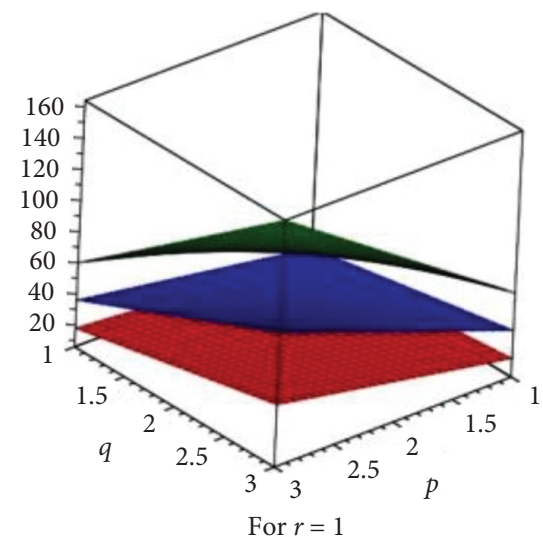

(c)

FIgURE 6: Comparison of IRR, $\mathrm{IRR}_{t}$, and IRF of crystallographic structure $\mathrm{Cu}_{2} \mathrm{O}[p, q, r]$.

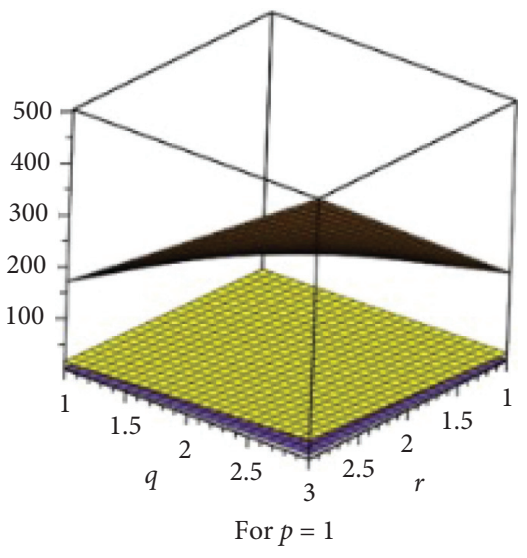

(a)

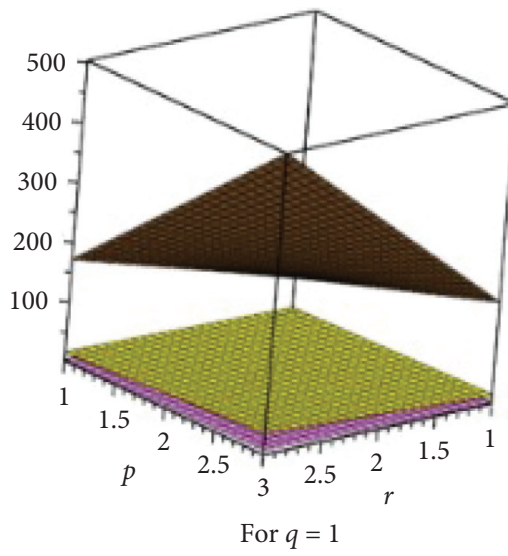

(b)

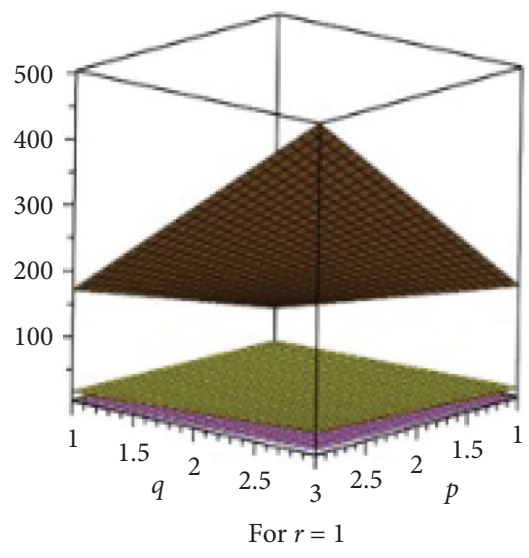

(c)

Figure 7: Comparison of IRL, $\mathrm{IRD}_{1}$, and IRGA for crystallographic structure $\mathrm{Cu}_{2} \mathrm{O}[p, q, r]$.

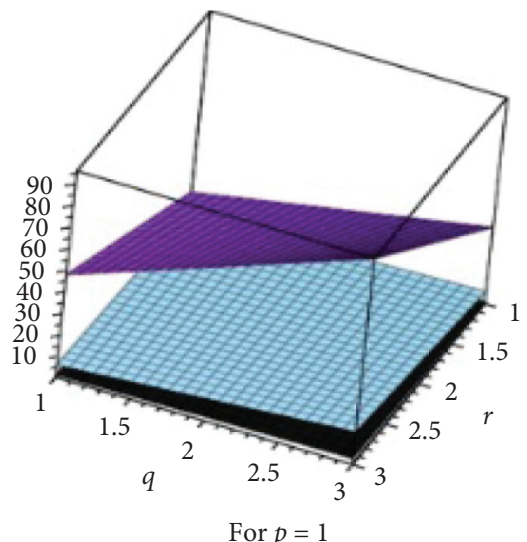

(a)

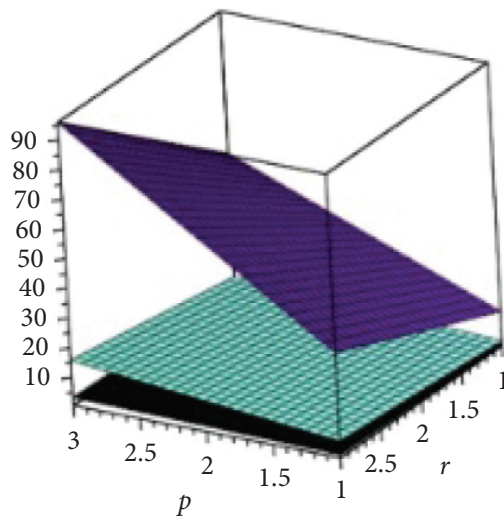

For $q=1$

(b)

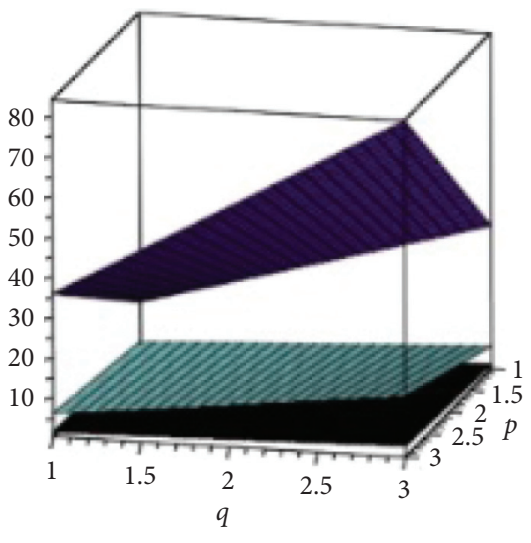

For $r=$

(c)

FIGURE 8: Comparison of IRA, IRB, and IRDIF for crystallographic structure $\mathrm{Cu}_{2} \mathrm{O}[p, q, r]$. 


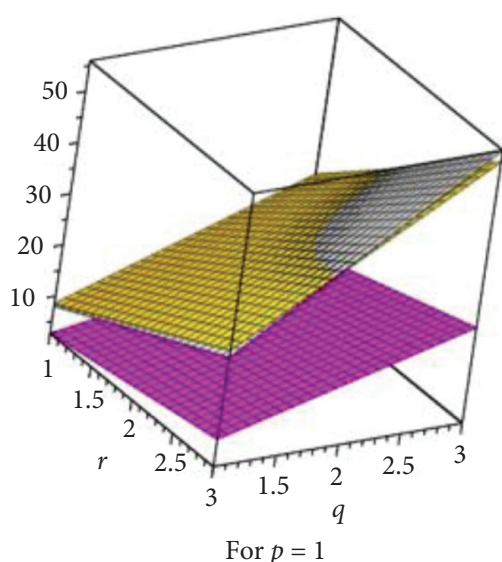

(a)

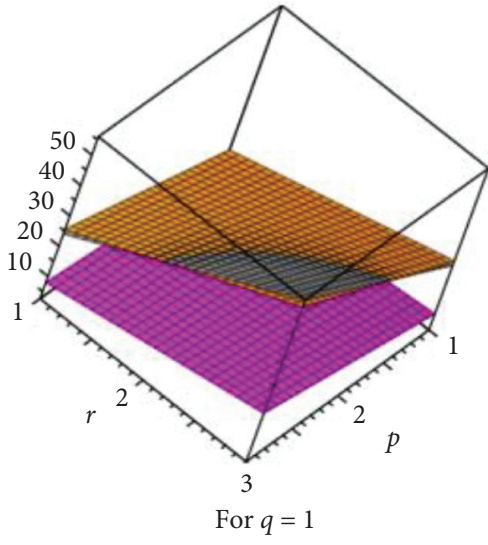

(b)

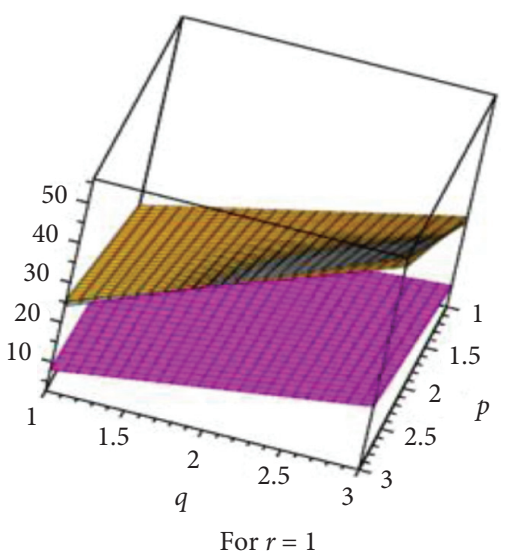

(c)

FIgURE 9: Comparison of IRLU, IRLA, and IRLF for crystallographic structure $\mathrm{Cu}_{2} \mathrm{O}[p, q, r]$.

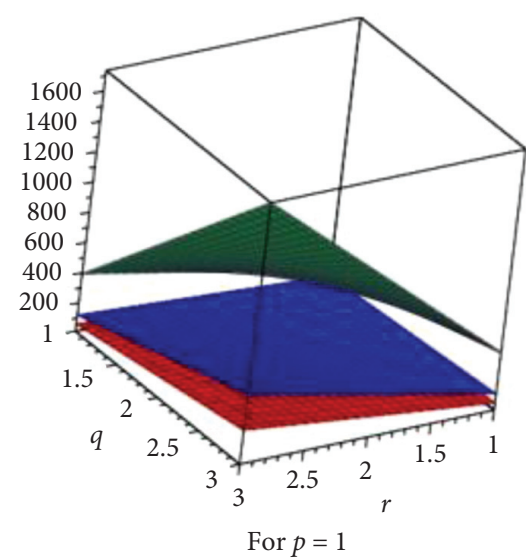

(a)

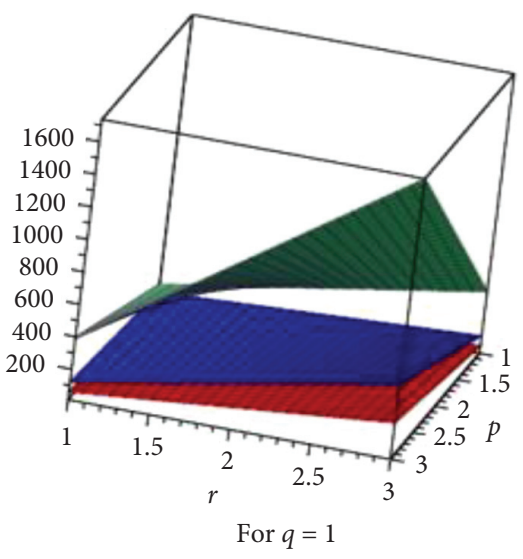

(b)

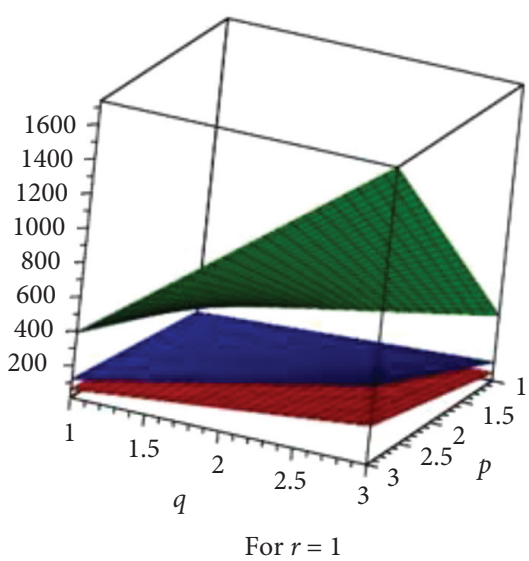

(c)

FiguRe 10: Comparison of IRR, IRR , and IRF for crystallographic structure of titanium difluoride $T_{i} F_{2}[p, q, r]$.

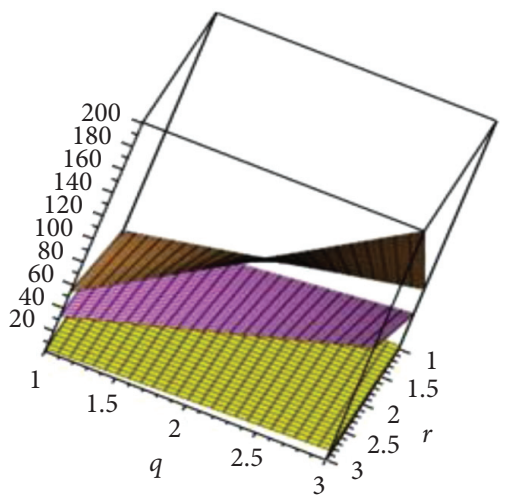

For $p=1$

(a)

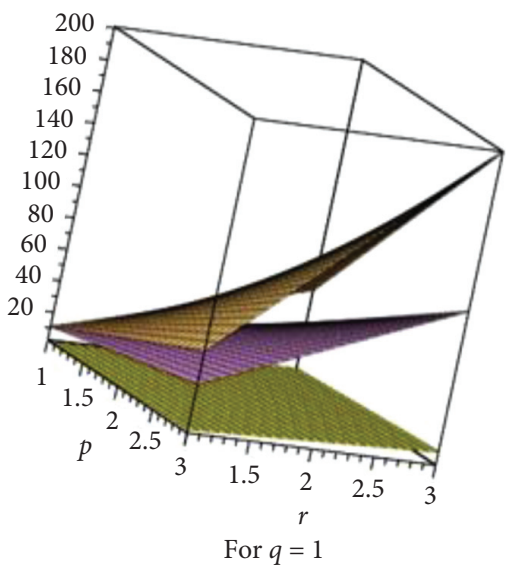

(b)

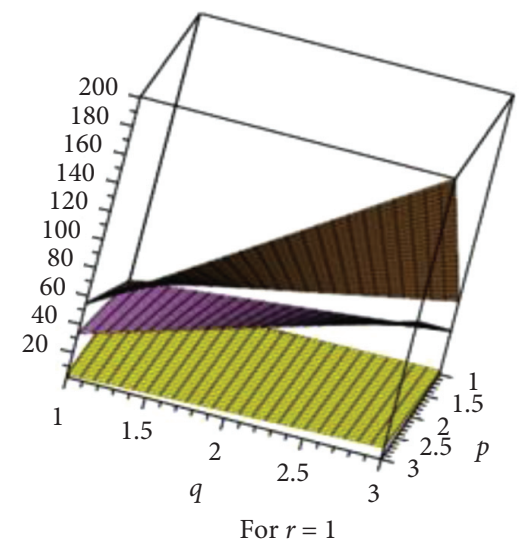

(c)

FIGURE 11: Comparison of IRL, $\operatorname{IRD}_{1}$, and IRGA for crystallographic structure of titanium difluoride $T_{i} F_{2}[p, q, r]$. 


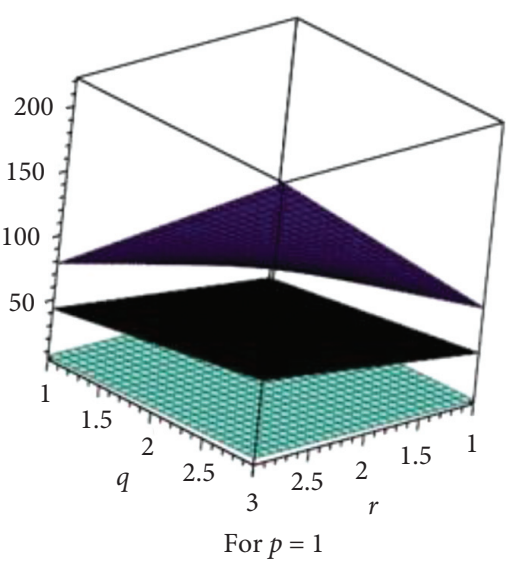

(a)

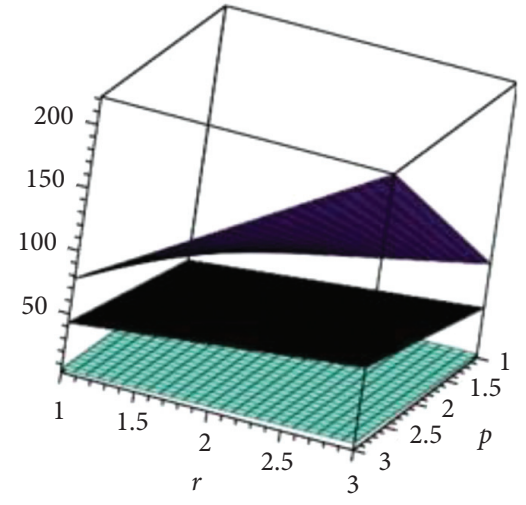

For $q=1$

(b)

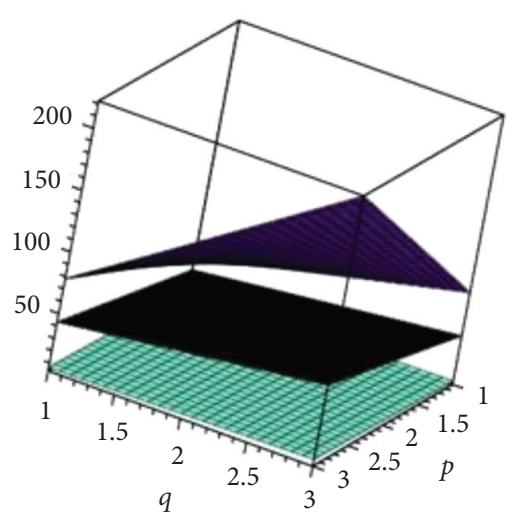

For $r=1$

(c)

FIgURE 12: Comparison of IRA, IRB, and IRDIF for crystallographic structure of titanium difluoride $T_{i} F_{2}[p, q, r]$.

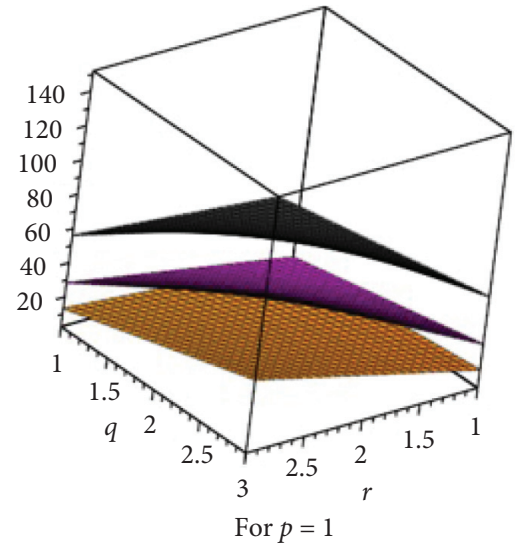

(a)

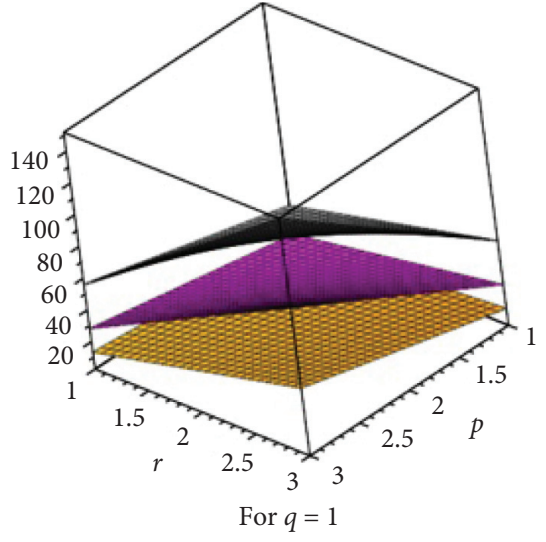

(b)

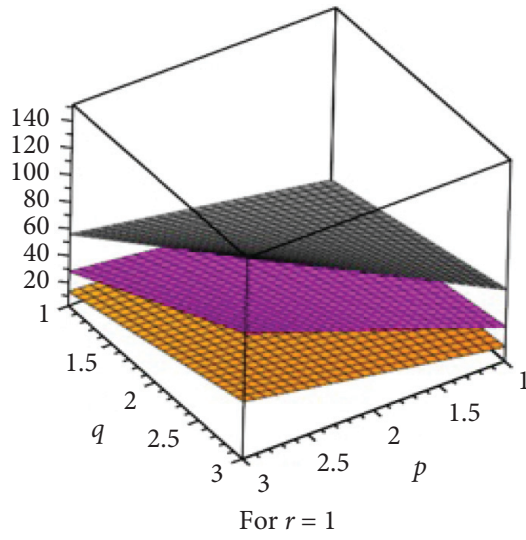

(c)

Figure 13: Comparison of IRLU, IRLA, and IRLF for crystallographic structure of titanium difluoride $T_{i} F_{2}[p, q, r]$.

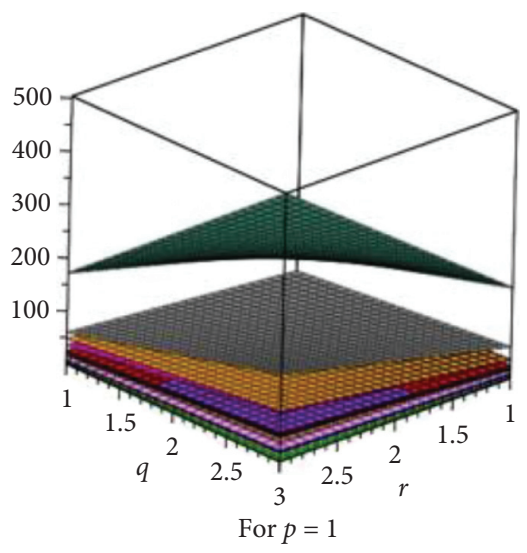

(a)

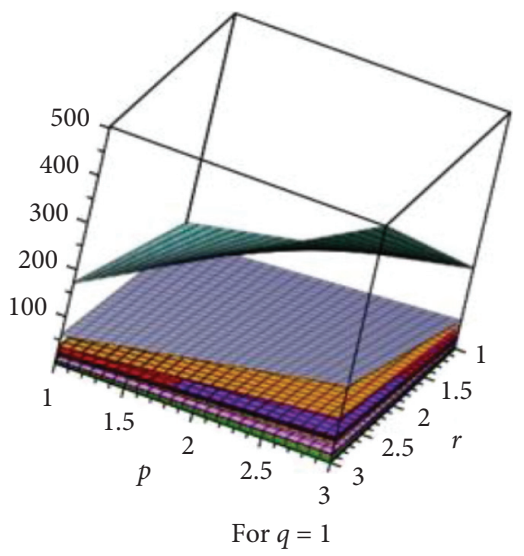

(b)

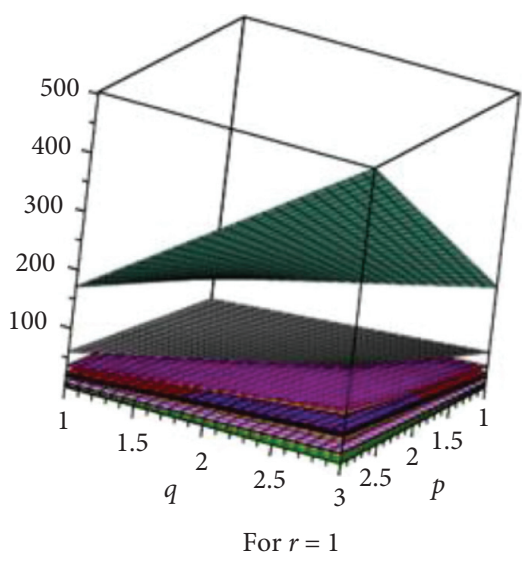

(c)

FIgURE 14: The comparison of all irregularity indices for crystallographic structure $\mathrm{Cu}_{2} \mathrm{O}[p, q, r]$. 


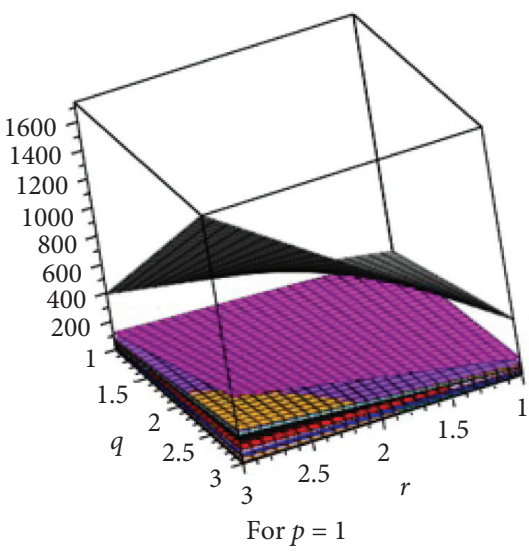

(a)

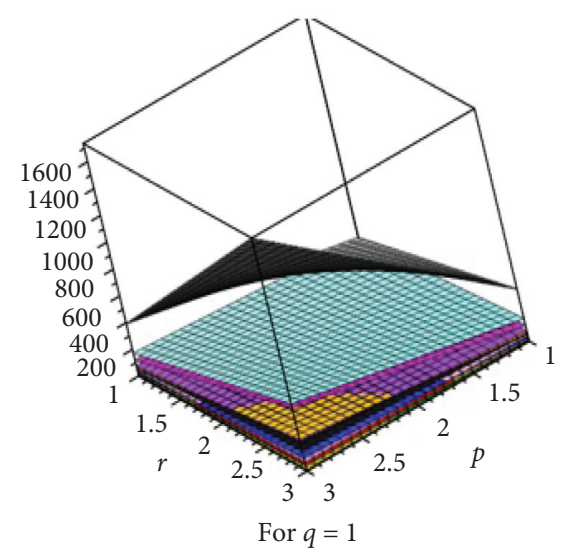

(b)

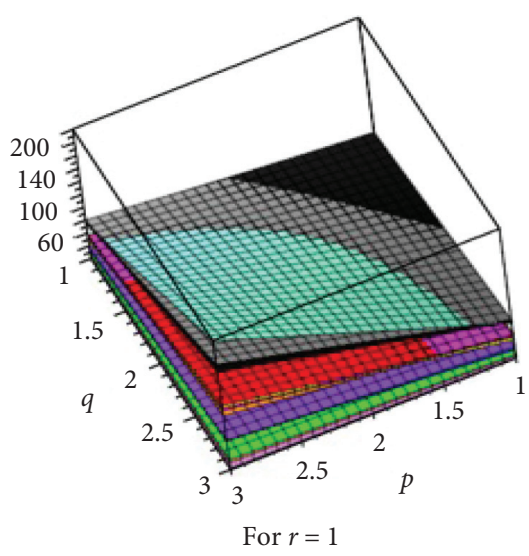

(c)

FIgURE 15: The comparison of all irregularity indices for crystallographic structure of titanium difluoride $T_{i} F_{2}[p, q, r]$.

Example 4. Let $T_{i} F_{2}[4,1,2]$ be the crystallographic structure of titanium difluoride in Figure 5. Then,

$$
\begin{aligned}
\operatorname{IRLU}\left(T_{i} F_{2}[4,1,2]\right) & =136, \\
\operatorname{IRLA}\left(T_{i} F_{2}[4,1,2]\right) & =40, \\
\operatorname{IRLF}\left(T_{i} F_{2}[4,1,2]\right) & =84.8528137423857, \\
\operatorname{IRDIF}\left(T_{i} F_{2}[4,1,2]\right) & =294 .
\end{aligned}
$$

\section{Comparisons and Discussion}

The main motivation comes from the fact that graphs of the irregularity indices show close accurate results about properties like entropy, standard enthalpy, vaporization, and acentric factors of octane isomers [8]. Irregularity indices may help to measure the chemical, biological, and nano properties which are widely popular in developing areas.

Through the means of a graph structural analysis and derivation, we compute some irregularity measures of crystallographic structure of molecules $\mathrm{Cu}_{2} \mathrm{O}[p, q, r]$ and the crystallographic structure of titanium difluoride of $T_{i} F_{2}[p, q, r]$. Similar works have been done in $[12,13,17]$. From Tables 2-9, we can easily see that all indices are in increasing order as the values of $p, q$, and $r$. On the other hand, indices showed higher values for $\mathrm{Cu}_{2} \mathrm{O}[p, q, r]$ and $T_{i} F_{2}[p, q, r]$. The graphical representations of irregularity indices of $\mathrm{Cu}_{2} \mathrm{O}[p, q, r]$ and $T_{i} F_{2}[p, q, r]$ are depicted in Figures 6-13 for certain values of $p, q$, and $r$. Moreover, we presented the comparison of all irregularity indices in Figures 14 and 15 for $\mathrm{Cu}_{2} \mathrm{O}[p, q, r]$ and $T_{i} F_{2}[p, q, r]$, respectively.

\section{Conclusion}

In this paper, we studied popular crystallographic structure of molecules and also applied analytical methods to compute the irregularity measures for crystallographic structure of molecules $\mathrm{Cu}_{2} \mathrm{O}[p, q, r]$ and crystallographic structure of titanium difluoride of $T_{i} F_{2}[p, q, r]$.

\section{Data Availability}

The data used to support the findings of this study are included within the article.

\section{Conflicts of Interest}

The authors declare that they have no conflicts of interest.

\section{References}

[1] N. Trinajsti, Chemical Graph Theory, CRC Press, Boca Raton, FL, USA, 1992.

[2] C. Wang, S. Wang, and B. Wei, "Cacti with extremal PI index," Transactions on Combinatorics, vol. 5, no. 4, pp. 1-8, 2016.

[3] D. B. West, R. Todeschini and V. Consonni, "An introduction to graph theory," Handbook of Molecular Descriptors, Prentice-Hall, Wiley, Weinheim, Germany, 1996.

[4] S. C. Basak, D. Mills, M. M. Mumtaz, and K. Balasubramanian, "Use of topological indices in predicting aryl hydrocarbon receptor binding potency of dibenzofurans: a hierarchical qsar approach," Indian Journal Chemistry, vol. 42A, pp. 1385-1391, 2003.

[5] I. Garcia, Y. Fall, and G. Gomez, "Using topological indices to predict anti-Alzheimer and anti-parasitic GSK-3 inhibitors by multi-target QSAR in silico screening," Molecules, vol. 15, pp. 5408-5422, 2010.

[6] M. Husin, R. Hasni, N. Arif, and M. Imran, "On topological indices of certain families of nanostar dendrimers," Molecules, vol. 21, no. 7, p. 821, 2016.

[7] T. Réti and A. Ali, "On the variance-type graph irregularity measures," Communications in Combinatorics and Optimization, vol. 5, pp. 169-178, 2020.

[8] T. Reti, R. Sharfdini, A. Dregelyi-Kiss, and H. Hagobin, "Graph irregularity indices used as molecular discriptors in QSPR studies," MATCH Communications in Mathematical and in Computer Chemistry, vol. 79, pp. 509-524, 2018.

[9] M. Saheli, H. Saati, and A. R. Ashrafi, Optoelectronics and Advanced Materials-Rapid Communications, vol. 4, p. 896, 2010.

[10] J. Zhang, J. Liu, Q. Peng, X. Wang, and Y. Li, "Nearly monodisperse $\mathrm{Cu}_{2} \mathrm{O}$ and $\mathrm{nCuO}$ anospheres, preparation and 
applications for sensitive gas sensors," Chemistry of Materials, vol. 18, no. 4, pp. 867-871, 2006.

[11] K. Chen, C. Sun, S. Song, and D. Xue, "Polymorphic crystallization of $\mathrm{Cu}_{2} \mathrm{O}$ compound," CrystEngComm Journal, vol. 16, pp. 52-57, 2014.

[12] Z. Iqbal, A. Aslam, M. Ishaq, and M. Aamir, "Characteristic study of irregularity measures of some nanotubes," Canadian Journal of Physics, vol. 97, no. 10, pp. 1125-1132, 2019.

[13] W. Gao, M. Aamir, Z. Iqbal, M. Ishaq, and A. Aslam, "On irregularity measures of some dendrimers structures," Mathematics, vol. 7, no. 3, p. 271, 2019.

[14] Z. Hussain, Sh. Rafique, M. Munir et al., "Irregularity molecular descriptors of hourglass, jagged-rectangle, and triangular benzenoid systems," Processes, vol. 7, no. 7, p. 413, 2019.

[15] Z. Iqbal, M. Ishaq, A. Aslam, M. Aamir, and W. Gao, "The measure of irregularities of nanosheets," Open Physics, vol. 18, no. 1, pp. 419-431, 2020.

[16] J. Zheng, S. Akhter, Z. Iqbal et al., "Irregularity measures of subdivision vertex-edge join of Graphs," Journal of Chemistry, vol. 2021, Article ID 6673221, 12 pages, 2021.

[17] H. Abdo, D. Dimitrov, and W. Gao, "On the irregularity of some molecular structures," Canadian Journal of Chemistry, vol. 95, no. 2, pp. 174-183, 2017.

[18] W. Gao, M. Younas, A. Farooq, A. Mahboob, and W. Nazeer, "M-polynomials and degree-based topological indices of the crystallographic structure of molecules," Biomolecules, vol. 8, no. 4, p. 107, 2018.

[19] S. Yousaf and A. A. Bhatti, "On the extremal total irregularity index of $n$-vertex trees with fixed maximum degree," Communications in Combinatorics and Optimization, vol. 6, pp. 113-121, 2021.

[20] G. Chartrand, P. Erdos, and O. R. Oellermann, "How to define an irregular graph," The College Mathematics Journal, vol. 19, no. 1, pp. 36-42, 1988.

[21] D. B. West, Introduction to Graph Theory, Prentice-Hall, Upper Saddle River, NJ, USA, 2001.

[22] M. O. Albertson, "The irregularity of a graph," Ars Combinatoria, vol. 46, pp. 219-225, 1997.

[23] A. Jahanbani, "Albertson energy and Albertson-Estrada index of graphs," Journal of Linear and Topological Algebra, vol. 8, pp. 11-24, 2019.

[24] H. Abdo, S. Brandt, and D. Dimitrov, "The total irregularity of a graph," Discrete Mathematics \& Theoretical Computer Science, vol. 16, pp. 201-206, 2014.

[25] I. Gutman, "Topological indices and irregularity measures," Journals Bulletin, vol. 8, pp. 469-475, 2018.

[26] B. Horoldagva, L. Buyantogtokh, S. Dorjsembe, and I. Gutman, "Maximum sizeof maximally irregular graphs," MATCH Communications in Mathematical and in Computer Chemistry, vol. 76, pp. 81-98, 2016.

[27] F. Liu, Z. Zhang, and J. Meng, "The size of maximally irregular graphs and maximally irregular triangle-free graphs," Graphs and Combinatorics, vol. 30, no. 3, pp. 699-705, 2014.

[28] L. Collatz and U. Sinogowitz, "Spektren endlicher graphen," Abhandlungen aus dem Mathematischen Seminar der Universitat Hamburg, vol. 21, pp. 63-77, 1957.

[29] F. A. Cotton, G. Wilkinson, C. A. Murillo, and M. Bochmann, Advanced Inorganic Chemistry, John Wiley and Sons, Hoboken, NJ, USA, 1999. 